\title{
Multi-argument fuzzy measures on lattices of fuzzy sets
}

\author{
Elena E. Castiñeira ${ }^{\mathrm{a}}$, Tomasa Calvo ${ }^{\mathrm{b}}$, Susana Cubillo ${ }^{\mathrm{a}}$ \\ ${ }^{a}$ Department of Applied Mathematics, Technical University of Madrid (UPM), Spain \\ ${ }^{\mathrm{b}}$ Department of Computer Sciences, University of Alcalá de Henares (UAH), Spain
}

\begin{abstract}
A B S T R A C T
In this paper, we axiomatically introduce fuzzy multi-measures on bounded lattices. In particular, we make a distinction between four different types of fuzzy set multi-measures on a universe $X$, considering both the usual or inverse real number ordering of this lattice and increasing or decreasing monotonicity with respect to the number of arguments. We provide results from which we can derive families of measures that hold for the applicable conditions in each case.
\end{abstract}

\section{Introduction}

Modern measure theory began to take shape in the late nineteenth century. Stolz and Harnack in 1884 and Cantor in 1885 defined the measure of a bounded set of $\mathbb{R}$ and a bounded set of $\mathbb{R}^{n}$, respectively. These measures have the disadvantage that the measure of the union of two disjoint sets can be smaller than the sum of the measures of the sets. This problem was solved in the case of finite unions when Peano and Jordan in 1890 introduced the notion of measurable set. However, this was not completely satisfactory, because, for example, the set of rational numbers in a bounded interval is not measurable. These difficulties were overcome when Borel [7] extended the class of measurable sets establishing a model in which the measure is countably additive. Lebesgue [34] followed up Borel's theory and related it to integration theory. When a measure is bounded on $[0,1]$ and assigns the value 1 to the universal set, then not only can it be used to estimate the size of a set, but it also provides a model to measure the probability of an event occurring. The concept of probability measure was defined by Kolmogorov [32] in 1933.

E-mail addresses: ecastineira@fi.upm.es (E.E. Castiñeira), tomasa.calvo@uah.es (T. Calvo), scubillo@fi.upm.es (S. Cubillo).
More than fifty years after the establishment of classical measure theory, the additivity requirement was questioned, and less restrictive theories began to emerge. For instance, Choquet presented the theory of capacities in 1954 [13], which calls into question the classical concept of measure since capacities are increasing and continuous set functions. In the context of probability theory, the Dempster-Shafer or evidence theory $[16,48]$ was developed in the second half of the twentieth century considering belief measures, which substitute the additivity requirement with superadditivity, and plausibility measures, which use subadditivity. Possibility measures [56] which are special plausibility measures, and the associated necessity measures, which are special belief measures, arose in the context of fuzzy sets, introduced by Zadeh in $1965[54,18,19]$.

Fuzzy set theory provided a framework in which new models of measurement were developed, even for measuring vague concepts. Thus, Sugeno introduced the fuzzy measure and fuzzy integral in 1974 [49], Ralescu and Adams [45] generalized these concepts to the case that the value a fuzzy measure can be infinite, Kruse [33], Wang [52] and Liu [36] studied some structural characteristics of fuzzy measures and proved several convergence theorems for fuzzy integrals, whereas Bassanezi and Greco [2] studied the representability of functionals defined on fuzzy sets by fuzzy measures. Sugeno's theory was used in knowledge-based systems that require a subjective evaluation for a family of non-fuzzy events 
[49], but today's systems often require the subjective and objective evaluation for a class of fuzzy events. This motivated authors such as Klement [29] and Qiao [43] to investigate fuzzy measures defined on fuzzy sets. Moreover, the concept of fuzzy measure was generalized considering measures defined or with their values on general lattices. Noteworthy in this regard are investigations by Greco [22], Qiao [44], Klement and Weber [31], and Liu and Zhang [37].

Up to now, we have referred to measures as functions with only one argument. Nevertheless there are measurable properties, like similarity and inclusion measures [24,55], and overlap functions [8], that involve two elements, thus requiring functions with two arguments. Moreover, there are also properties that can refer to more than two or even a variable number of elements; for example, we might want to study the incompatibility of several sets or predicates in systems modeling with more than one source of information. Therefore, it is worthwhile building a broader measurement model that could be applied to a variable number of arguments. Different theoretical developments of multi-argument functions are reported in the literature, such as the thoroughly researched aggregation functions $[3,9,50,20]$. It is well-known that some aggregation functions can be seen as fuzzy integrals with respect to a system of fuzzy measures. This is the case of the arithmetic mean, which is the Choquet integral with respect to an additive and symmetric fuzzy measure. More recently, the class of basic generated universal fuzzy measures has been introduced by Mesiar et al. $[39,40]$. The integrals associated with this class of fuzzy measures include the generated OWA operators and the generated weighted means, which are examples of aggregation functions $[17,42,38]$. Also noteworthy in this context is another recent work by Mesiar and Komorniková on aggregation functions on bounded posets [41].

This paper, which further develops ideas first reported in [10], introduces a generalized model of fuzzy measures with multiple arguments defined on lattices, and it includes aggregation functions as a particular case. We have previously introduced and studied several measures in the field of fuzzy and Atanassov's sets, in particular contradiction measures $[12,14]$ and incompatibility measures [11]. In both cases, we defined a mathematical model for functions with just two arguments. Now, we again address the fuzzy concept of incompatibility, but this time as part of a multi-argument function model that captures our previous research. Additionally, and as an instrument for modeling other fuzzy concepts, such as compatibility and supplementarity, we address new measures.

From the viewpoint of their application, we might want to know, for example, how compatible and how supplementary a set of fixed criteria are in any multi-criteria decision problem $[4,23]$. Recently, Liu et al. showed that decision making is based on an inclusion measure function which is based on a positive evaluation [35]. Consequently, this approach determines a specific vie to interpret compatibility. Additionally, lattice theory has been applied in mathematical morphology including image processing $[53,46]$. In particular, fuzzy lattices have been used in clustering and classification algorithms and in fuzzy lattice neurocomputing [25]. Fuzzy lattice theory has the ability to model uncertain information and to combine different types of data [27]. Also, it has redesigned fuzzy inference systems $[27,28]$, therefore fuzzy lattice reasoning is applied in computational intelligence [26]. Compatibility measures are a key concern in knowledge representation, as they are very useful in environments where information is unclear or possibly distorted as it comes from different hubs or sources. Notice that the concept of compatibility in fuzzy logic has been interpreted from different viewpoints. Some authors have looked at compatibility as the degree of inclusion, whereas others view it as the overlap between two sets, the degree of equality, similarity, etc. In this paper, we will interpret compatibility as a direct extension of the classical case, where two sets are compatible if their intersection is not empty.

The paper is organized as follows. Section 2 defines the multiargument fuzzy measure on a lattice, and puts forward some examples and properties. Section 3 focuses on two types of multi-measures on the lattice $[0,1]^{X}$ with the usual real number ordering, that is, compatibility measures and supplementarity measures. Section 4 addresses another two types of multi-measures on the lattice $[0,1]^{X}$ with reverse real number ordering, unsupplementarity and incompatibility multi-measures. Section 5 shows some basic relations among multi-measures. Finally, Section 6 presents an example of application, and the paper ends with the conclusions.

\section{Multi-argument fuzzy measures on lattices}

Let $\mathcal{L}=\left(L, \leqslant_{L}, 0_{L}, 1_{L}\right)$ be a bounded lattice $[5,6]$ whose minimum and maximum elements are denoted by $0_{L}$ and $1_{L}$, respectively. For each $n \in \mathbb{N}$, let us consider the set

$L^{n}=\left\{\left(a_{1}, \ldots, a_{n}\right) \mid a_{i} \in L, \quad \forall i \in\{1, \ldots, n\}\right\}$

and the order relation $\leqslant_{L^{n}}$ induced by $\leqslant_{L}$, that is, given $\bar{a}=\left(a_{1}, \ldots, a_{n}\right), \bar{b}=\left(b_{1}, \ldots b_{n}\right) \in L^{n}$,

$\bar{a} \leqslant L^{n} \bar{b} \Longleftrightarrow a_{i} \leqslant L b_{i}, \quad \forall i \in\{1, \ldots, n\}$.

We have that $L^{n}$ with the order relation $\Sigma_{L^{n}}$ is also a bounded lattice, whose minimum element is $\left.0_{L^{n}}=\left(0_{L}, ., 2\right), 0_{L}\right)$ and whose maximum element is $\left.1_{L^{n}}=\left(1_{L}, n\right), 1_{L}\right)$. We say that $\mathcal{L}^{n}=\left(L^{n}, \leqslant L^{n}, 0_{L^{n}}, 1_{L^{n}}\right)$ is induced by $\mathcal{L}$. Moreover, if $\mathcal{L}$ is complete, then $\mathcal{L}^{n}$ is also complete.

We denote the bounded and complete lattice of real numbers $([0,1], \leqslant, 0,1)$, which is used throughout the paper, by $\mathfrak{\Im}$.

Definition 2.1. Given a bounded lattice $\mathcal{L}=\left(L, \leqslant L, 0_{L}, 1_{L}\right)$, the lattice $\mathcal{L}^{n}$ induced by $\mathcal{L}$, for a fixed $n \in \mathbb{N}$, and the lattice of real numbers $\Im$, a map $M: L^{n} \rightarrow[0,1]$ is said to be a fuzzy measure on $\mathcal{L}^{n}$ or a fuzzy n-measure on $\mathcal{L}$ (or on $L$ ), if it satisfies:

(i) $M\left(0_{L^{n}}\right)=0$ and $M\left(1_{L^{n}}\right)=1$ (boundary conditions).

(ii) $M$ is increasing with respect to the orders of the lattices $\mathcal{L}^{n}$ and $\mathfrak{\Im}$, that is, if $\bar{a}, \bar{b} \in L^{n}$ such that $\bar{a} \leqslant_{L^{n}} \bar{b}, M(\bar{a}) \leqslant M(\bar{b})$ holds (monotony condition).

If $n=1$ then $\mathrm{M}$ is said to be a fuzzy measure on $\mathcal{L}$ (or on $L$ ).

We can extend the definition of fuzzy measure by considering any number of arguments as follows.

Definition 2.2. Let $\mathcal{L}=\left(L, \leqslant_{L}, 0_{L}, 1_{L}\right)$ be a bounded lattice and let $\mathcal{L}^{n}$ be the lattice induced by $\mathcal{L}$, for each $n \in \mathbb{N}$. A map $M: \bigcup_{n \in \mathbb{N}} L^{n} \rightarrow[0,1]$ is said to be a multi-argument fuzzy measure or fuzzy multi-measure on $\mathcal{L}$ (or on $L$ ) if, for each $n \in \mathbb{N}$, the function $M$ restricted to $L^{n},\left.M\right|_{L^{n}}$, is a fuzzy $n$-measure. Moreover,

(iii) $M$ is increasing with respect to the argument $n$ or $n$-increasing if $M\left(a_{1}, \ldots, a_{n}\right) \leqslant M\left(a_{1}, \ldots, a_{n}, a_{n+1}\right)$ holds for all $n \in \mathbb{N}$ and for all $a_{1}, \ldots, a_{n}, a_{n+1} \in L$.

(iv) $M$ is decreasing with respect to the argument $n$ or $n$-decreasing if $M\left(a_{1}, \ldots, a_{n}\right) \geqslant M\left(a_{1}, \ldots, a_{n}, a_{n+1}\right)$ holds for all $n \in \mathbb{N}$ and for all $a_{1}, \ldots, a_{n}, a_{n+1} \in L$.

Remark 2.3. If $M$ is a fuzzy multi-measure on $\mathcal{L}$, note that:

1. $M$ is $n$-increasing (or dimension increasing) if and only if $M\left(a_{1}, \ldots, a_{n}\right) \leqslant M\left(a_{1}, \ldots, a_{n}, b_{1}, \ldots, b_{m}\right)$ holds for all $\left(a_{1}, \ldots, a_{n}\right)$ $\in L^{n},\left(b_{1}, \ldots, b_{m}\right) \in L^{m}$ and $n, m \in \mathbb{N}$. 
2. $M$ is $n$-decreasing (or dimension decreasing) if and only if $M\left(a_{1}, \ldots, a_{n}\right) \geqslant M\left(a_{1}, \ldots, a_{n}, b_{1}, \ldots, b_{m}\right)$ holds for all $\left(a_{1}, \ldots, a_{n}\right)$ $\in L^{n},\left(b_{1}, \ldots, b_{m}\right) \in L^{m}$ and $n, m \in \mathbb{N}$.

Example 2.4. Let $X$ be a non-empty and finite set, and let $\mathcal{P}(X)$ denote the set of all subsets of $X$, that is, the power set of $X$. Consider the bounded lattice $(\mathcal{P}(X), \subseteq \emptyset, X)$, which is, in fact, a Boolean algebra, and let us define two multi-argument fuzzy measures on $\mathcal{P}(X)$.

(a) Let $M_{I}: \bigcup_{n \in \mathbb{N}} \mathcal{P}(X)^{n} \rightarrow[0,1]$ be the map defined for each $\left(A_{1}, \ldots, A_{n}\right) \in \mathcal{P}(X)^{n}$ as

$M_{I}\left(A_{1}, \ldots, A_{n}\right)=\frac{\left|A_{1} \cap \cdots \cap A_{n}\right|}{|X|}$,

where $|A|$ means cardinal of the set $A$. Then $M_{I}$ satisfies:

(i) $M_{f}(\emptyset, \ldots, \emptyset)=0$ for all $n$-tuples of empty sets; and $M_{r}(-$ $X, \ldots, X)=1$ for all $n$-tuples of coordinates $X$.

(ii) For each $n \in \mathbb{N}, M_{I}\left(A_{1}, \ldots, A_{n}\right) \leqslant M_{I}\left(B_{1}, \ldots, B_{n}\right)$ holds provided $A_{i}, B_{i} \in \mathcal{P}(X)$ satisfy $A_{i} \subseteq B_{i}$ for each $i \in\{1, \ldots, n\}$.

(iii) $M_{r}\left(A_{1}, \ldots, A_{n}, A_{n+1}\right) \leqslant M_{I}\left(A_{1}, \ldots, A_{n}\right)$ for all $A_{1}, \ldots$, $A_{n}, A_{n+1} \in \mathcal{P}(X)$ and $n \in \mathbb{N}$.

Hence, $M_{I}$ is an $n$-decreasing multi-argument fuzzy measure on $\mathcal{P}(X) . M_{I}$ provides an estimate of the relative size, compared with the universal set, of the intersection of any finite family of subsets.

b) Let $M_{U}: \bigcup_{n \in \mathbb{N}} \mathcal{P}(X)^{n} \rightarrow[0,1]$ be the map defined for each $\left(A_{1}, \ldots, A_{n}\right) \in \mathcal{P}(X)^{n}$ as

$M_{U}\left(A_{1}, \ldots, A_{n}\right)=\frac{\left|A_{1} \cup \cdots \cup A_{n}\right|}{|X|}$.

Then, $M_{U}$ also satisfies axioms (i) and (ii) of fuzzy $n$-measure for each $n \in \mathbb{N}$ and, also, axiom (iii). Therefore $M_{U}$ is an $n$-increasing multi-argument fuzzy measure on $\mathcal{P}(X) . M_{U}$ provides an estimate of the relative size, compared with the universal set, of the union of any finite family of subsets.

Example 2.5. Any aggregation function is a fuzzy multi-measure on $\mathfrak{\Im}$. Indeed, recall that an aggregation function $[3,9,20,30]$ is a map $\mathcal{A}: \bigcup_{n \in \mathbb{N}}[0,1]^{n} \rightarrow[0,1]$ such that

1. $\mathcal{A}(0, \ldots, 0)=0$ and $\mathcal{A}(1, \ldots, 1)=1$.

2. $\mathcal{A}(a)=a$ for all $a \in[0,1]$.

3. For each $n \in \mathbb{N}, \mathcal{A}\left(a_{1}, \ldots, a_{n}\right) \leqslant \mathcal{A}\left(b_{1}, \ldots, b_{n}\right)$ holds provided $a_{i}$, $b_{i} \in[0,1]$ satisfy $a_{i} \leqslant b_{i}$ for all $i \in\{1, \ldots, n\}$.

Thus, the occurrence of symmetric aggregation functions suggests the following definition. We denote $S_{n}=\{\pi:\{1, \ldots, n\} \rightarrow$ $\{1, \ldots, n\} \mid \pi$ is a bijection $\}$, that is, $S_{n}$ is the set of permutations of $\{1, \ldots, n\}$.

Definition 2.6. A multi-argument fuzzy measure $M$ on a bounded lattice $\mathcal{L}=\left(L, \leqslant L, O_{L}, 1_{L}\right)$ is symmetric if, for each $n \in \mathbb{N}$, the function $\left.M\right|_{L^{n}}$ is symmetric, that is, $M\left(a_{1}, \ldots, a_{n}\right)=M\left(a_{\pi(1)}, \ldots, a_{\pi(n)}\right)$ holds for any $\pi \in S_{n}$ and for any $\left(a_{1}, \ldots, a_{n}\right) \in L^{n}$.

Example 2.7. The maps $M_{I}$ and $M_{U}$ defined in Example 2.4 are both symmetric fuzzy multi-measures on $\mathcal{P}(X)$.

Example 2.8. Let us illustrate the property of symmetry with some aggregation functions: (a) The functions Max, Min : $\bigcup_{n \in \mathbb{N}}[0,1]^{n} \rightarrow[0,1]$, defined as $\operatorname{Max}\left(a_{1}, \ldots, a_{n}\right)=\max \left\{a_{1}, \ldots, a_{n}\right\}$ and $\operatorname{Min}\left(a_{1}, \ldots, a_{n}\right)=$ $\min \left\{a_{1}, \ldots, a_{n}\right\}$ for each $\left(a_{1}, \ldots, a_{n}\right) \in[0,1]^{n}$, are symmetric fuzzy multi-measures on $\mathfrak{\Im}$.

(b) For each $k \in \mathbb{N} \backslash\{1\}$, let $\mathcal{A}_{k}: \bigcup_{n \in \mathbb{N}}[0,1]^{n} \rightarrow[0,1]$ be the function defined for each $\left(a_{1}, \ldots, a_{n}\right) \in[0,1]^{n}$ as

$$
\mathcal{A}_{k}\left(a_{1}, \ldots, a_{n}\right)=a_{1} \prod_{i=2}^{n} a_{i}^{k} .
$$

Then $\left\{\mathcal{A}_{k}\right\}_{k \in \mathbb{N} \backslash\{1\}}$ is a one-parameter family of non-symmetric fuzzy multi-measures on $\mathfrak{I}$.

(c) For each $i, k \in \mathbb{N} \backslash\{1\}$, let $\mathcal{A}_{i k}: \bigcup_{n \in \mathbb{N}}[0,1]^{n} \rightarrow[0,1]$ be a function defined, for each $\left(a_{1}, \ldots, a_{n}\right) \in[0,1]^{n}$, by

$$
\mathcal{A}_{i k}\left(a_{1}, \ldots, a_{n}\right)= \begin{cases}\prod_{j=1}^{n} a_{j} & \text { if } n \leqslant i, \\ a_{i}^{k} \prod_{\substack{j=1 \\ j \neq i}}^{n} a_{j} & \text { if } n>i .\end{cases}
$$

Then $\left\{\mathcal{A}_{i k}\right\}_{i, k \in \mathbb{N} \backslash\{1\}}$ is a two-parameter family of non-symmetric fuzzy multi-measures on $\mathfrak{\Im}$.

Other fuzzy n- or multi-measures can be constructed by composing a fuzzy n- or multi-measure with an appropriate function, as shown by the following result, which, although trivial, is useful for this purpose. To do this, we use the set $\mathcal{A}([0,1])=$ $\{\varphi:[0,1] \rightarrow[0,1] \mid \varphi$ is an increasing bijection $\}$.

Proposition 2.9. Let $\mathcal{L}=\left(L, \leqslant L, 0_{L}, 1_{L}\right)$ be a bounded lattice. If $M$ is a fuzzy measure on $\mathcal{L}^{n}$, or a fuzzy multi-measure on $\mathcal{L}$, then for all $\varphi \in \mathcal{A}([0,1])$, the composition $\varphi \circ M$ is a fuzzy measure on $\mathcal{L}^{n}$ or, respectively, a fuzzy multi-measure on $\mathcal{L}$.

According to the following result whose proof is straightforward, aggregation functions defined on $[0,1]$ in the same way as on any lattice provide two ways to construct fuzzy multi-measures from a given fuzzy measure. One way is to aggregate measures of lattice elements, whereas the other is to measure the aggregation of lattice elements.

Proposition 2.10. Let $\mathcal{L}=\left(L, \leqslant_{L}, 0_{L}, 1_{L}\right)$ be a bounded lattice and $m: L \rightarrow[0,1]$ be a fuzzy measure on $\mathcal{L}$, it holds that:

1. If $\mathcal{A}$ is a fuzzy multi-measure on $\Im$, then $M_{\mathcal{A}}: \bigcup_{n \in \mathbb{N}} L^{n} \rightarrow[0,1]$, defined for each $\left(a_{1}, \ldots, a_{n}\right) \in L^{n}$ by

$$
M_{\mathcal{A}}\left(a_{1}, \ldots, a_{n}\right)=\mathcal{A}\left(m\left(a_{1}\right), \ldots, m\left(a_{n}\right)\right),
$$

is a fuzzy multi-measure on $\mathcal{L}$. Moreover, if $\mathcal{A}$ is an aggregation function, then $M_{\mathcal{A}}(a)=m(a)$ for all $a \in L$.

2. If $\mathcal{F}: \bigcup_{n \in \mathbb{N}} L^{n} \rightarrow L$ is a multi-argument function such that $\mathcal{F}\left(0_{L}, \ldots, 0_{L}\right)=0_{L}, \mathcal{F}\left(1_{L}, \ldots, 1_{L}\right)=1_{L}$ and $\mathcal{F}\left(a_{1}, \ldots, a_{n}\right) \leqslant \mathcal{F}\left(b_{1}, \ldots\right.$, $\left.b_{n}\right)$, whenever $a_{i} \leqslant{ }_{L} b_{i}$ for each $i \in\{1, \ldots, n\}$ and for all $n \in \mathbb{N}$, then the multi-argument function $M_{\mathcal{F}}: \bigcup_{n \in \mathbb{N}} L^{n} \rightarrow[0,1]$, defined for each $\left(a_{1}, \ldots, a_{n}\right) \in L^{n}$ by

$$
M_{\mathcal{F}}\left(a_{1}, \ldots, a_{n}\right)=m\left(\mathcal{F}\left(a_{1}, \ldots, a_{n}\right)\right),
$$

is a fuzzy multi-measure on $\mathcal{L}$. Moreover, if $\mathcal{F}(a)=$ a for all $a \in L$, then $M_{\mathcal{F}}(a)=m(a)$ for all $a \in L$.

Example 2.11. Let us illustrate the methods in Proposition 2.10 with some examples.

(a) If $\mathcal{N}_{n}$ denotes the set of the first $n$ natural numbers, consider the particular case $\mathcal{N}_{3}=\{1,2,3\}$ and the power set of $\mathcal{N}_{3}$ with the order $\subseteq$, thus $\left(\mathcal{P}\left(\mathcal{N}_{3}\right), \subseteq, \emptyset, \mathcal{N}_{3}\right)$ is a bounded lattice. Let $v: \mathcal{P}\left(\mathcal{N}_{3}\right) \rightarrow[0,1]$ be the map defined according to the arrange- 
ment shown in Fig. 1. Note that $v$ is a discrete fuzzy measure $[17,21,42]$ since such a measure is defined as a set function $m$ from $\mathcal{P}\left(\mathcal{N}_{n}\right)$ on $[0,1]$ that satisfies $m(\emptyset)=0, m\left(\mathcal{N}_{n}\right)=1$ and is increasing, that is, $m(A) \leqslant m(B)$ whenever $A \subset B$.

From here, by means of an aggregation function $\mathcal{A}$, we can define the fuzzy multi-measure $M_{\mathcal{A}}: \bigcup_{n \in \mathbb{N}} \mathcal{P}\left(\mathcal{N}_{3}\right)^{n} \rightarrow[0,1]$ defined for each $\left(A_{1}, \ldots, A_{n}\right) \in \mathcal{P}\left(\mathcal{N}_{3}\right)^{n}$ by

$M_{\mathcal{A}}\left(A_{1}, \ldots, A_{n}\right)=\mathcal{A}\left(v\left(A_{1}\right), \ldots, v\left(A_{n}\right)\right)$.

Let us examine different types of fuzzy multi-measures defined by several aggregation functions.

1. If $\mathcal{A}=\operatorname{Max}$ or $\mathcal{A}=$ Min, then $M_{\mathcal{A}}$ is a symmetric fuzzy multimeasure on $\mathcal{P}\left(\mathcal{N}_{3}\right)$.

2. For each $k \in \mathbb{N} \backslash\{1\}$, let $\mathcal{A}=\mathcal{A}_{k}$ given in Example 2.8, where $\mathcal{A}_{k}\left(a_{1}, \ldots, a_{n}\right)=a_{1} \prod_{j \neq 1} a_{j}^{k}$ for each $\left(a_{1}, \ldots, a_{n}\right) \in[0,1]^{n}$. Then $M_{\mathcal{A}_{k}}$ is a non-symmetric fuzzy multi-measure on $\mathcal{P}\left(\mathcal{N}_{3}\right)$.

b) The mappings $M_{I}$ and $M_{U}$ given in Example 2.4 can be obtained by means of the map $m: \mathcal{P}(X) \rightarrow[0,1]$ defined by $m(A)=\frac{|A|}{|X|}$ for all $A \in \mathcal{P}(X)$, that is, a fuzzy measure on $\mathcal{P}(X)$. Indeed:

1. Consider the multi-argument function $\mathcal{I}: \bigcup_{n \in \mathbb{N}} \mathcal{P}(X)^{n} \rightarrow \mathcal{P}(X)$ defined for each $\left(A_{1}, \ldots, A_{n}\right) \in \mathcal{P}(X)^{n}$ by $\mathcal{I}\left(A_{1}, \ldots, A_{n}\right)=$ $A_{1} \cap \ldots \cap A_{n}$. Then,

$$
M_{I}\left(A_{1}, \ldots, A_{n}\right)=m\left(\mathcal{I}\left(A_{1}, \ldots, A_{n}\right)\right)=M_{I}\left(A_{1}, \ldots, A_{n}\right)
$$

holds for all $\left(A_{1}, \ldots, A_{n}\right) \in \mathcal{P}(X)^{n}$.

2. Consider the multi-argument function $\mathcal{U}: \bigcup_{n \in \mathbb{N}} \mathcal{P}(X)^{n} \rightarrow \mathcal{P}(X)$ defined for each $\left(A_{1}, \ldots, A_{n}\right) \in \mathcal{P}(X)^{n}$ by $\mathcal{U}\left(A_{1}, \ldots, A_{n}\right)=$ $A_{1} \cup \ldots \cup A_{n}$. Then,

$$
M_{\mathcal{U}}\left(A_{1}, \ldots, A_{n}\right)=m\left(\mathcal{U}\left(A_{1}, \ldots, A_{n}\right)\right)=M_{U}\left(A_{1}, \ldots, A_{n}\right)
$$

holds for all $\left(A_{1}, \ldots, A_{n}\right) \in \mathcal{P}(X)^{n}$.

In what follows, we study the particular instance of multi-measures on lattices of fuzzy sets. Given $X \neq \emptyset$, we consider the set of membership functions of the fuzzy sets on $X,[0,1]^{X}=$ $\{\mu: X \rightarrow[0,1]\}$, with an order relation $\preceq$ such that $\mathcal{L}=$ $\left([0,1]^{X}, \preceq, \mu_{\wedge}, \mu_{\vee}\right)$ is a bounded lattice, where $\mu_{\wedge}$ and $\mu_{\vee}$ denote the minimum and maximum elements, respectively. For each $n \in \mathbb{N}$, if $\square_{n}$ denotes $[0,1]^{n}, \mathcal{L}$ induces the bounded lattice $\mathcal{L}^{n}=\left(\square_{n}^{X}, \swarrow_{n}, \bar{\mu}_{\wedge}, \bar{\mu}_{\vee}\right)$ as $\left.\rrbracket_{n}^{X}=\left([0,1]^{n}\right)^{X}=[0,1]^{X} \times{ }^{n}\right) \times[0,1]^{X}$. Thus, a fuzzy multi-measure on $\mathcal{L}$ is a multi-argument function $M: \bigcup_{n \in \mathbb{N}} \mathbb{N}_{n}^{X} \rightarrow[0,1]$ such that: i) $M\left(\bar{\mu}_{\wedge}\right)=0$ and $M\left(\bar{\mu}_{\vee}\right)=1$; and ii) $M(\bar{\mu}) \leqslant M(\bar{\sigma})$ holds for all $\bar{\mu}, \bar{\sigma} \in \square_{n}^{X}$ such that $\bar{\mu} \preceq_{n} \bar{\sigma}$.
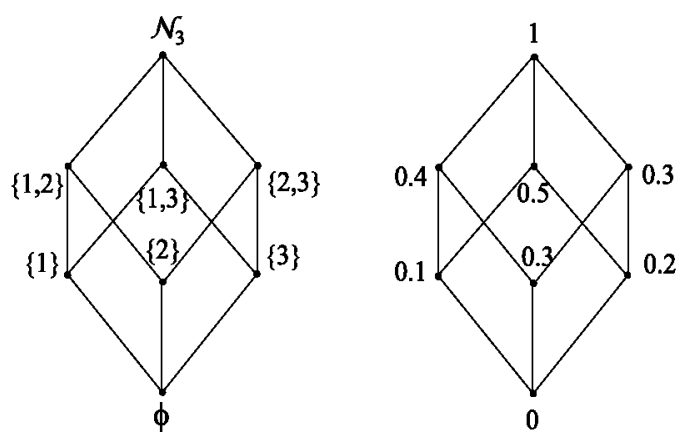

Fig. 1. Lattice $\left(\mathcal{P}\left(\mathcal{N}_{3}\right), \subseteq, \emptyset, \mathcal{N}_{3}\right)$, on the left side, and values of map $v$, on the right side.

\section{Fuzzy multi-measures on lattice $\left([0,1]^{X}, \leqslant\right)$}

In this section, we deal with multi-measures on $[0,1]^{X}$ with the order induced by the usual order of real numbers, which is denoted in the same way, that is, given $\mu, \sigma \in[0,1]^{X}, \mu \leqslant \sigma$ if and only if $\mu(x) \leqslant \sigma(x)$ for all $x \in X$. In this case, $\mu_{\wedge}=\mu_{\emptyset}$ and $\mu_{\vee}=\mu_{X}$, where $\mu_{\emptyset}(x)=0$ and $\mu_{X}(x)=1$ for all $x \in X$. Thus $\mathcal{L}=\left([0,1]^{X}, \leqslant, \mu_{\emptyset}, \mu_{X}\right)$, and we denote concisely $\left([0,1]^{X}, \leqslant\right)$.

Let us look at two types of multi-measures on $\left([0,1]^{X}, \leqslant\right)$ : multimeasures that evaluate how compatible a set of fuzzy sets is and multi-measures that evaluate how supplementary the set is. Remember that, in classical logic, two statements are compatible if they can both be true at the same time. As we can identify a statement on a universe $X$ with the set of elements of $X$ that satisfy that statement, we can translate this concept to set theory: $A, B \subset X$ are compatible if $A \cap B \neq \emptyset$. On the other hand, supplementarity can, in a sense, be understood as a symmetric property of incompatibility: $A$ and $B$ are supplementary if $A \cup B=X$. These concepts are extended to the fuzzy set framework and studied in the following sections.

\subsection{Compatibility multi-measures on fuzzy sets}

In order to define compatible fuzzy sets, we need a function that models the intersection of fuzzy sets, that is, a t-norm. Remember that a $t$-norm $[1,30,47]$ is a binary aggregation function $T$ on the unit interval $[0,1]$, which is commutative, associative, monotone increasing with respect to the usual order on the real line, and whose neutral element is 1 . Some of the main t-norms are the so-called Lukasiewicz, product and minimum t-norms, which are defined, respectively, by $T_{L}(a, b)=\max \{0, a+b-1\}, T_{P}(a, b)=a \cdot b$ and $\operatorname{Min}(a, b)=\min \{a, b\}$, for each $(a, b) \in[0,1]^{2}$.

Since a t-norm $T$ is associative and commutative, then it also defines a symmetric multi-argument function on [0,1], which is also denoted by $T$, and thus a symmetric fuzzy multi-measure on $[0,1]$, as follows: $T: \bigcup_{n \in \mathbb{N}}[0,1]^{n} \rightarrow[0,1]$ such that (1) $T(a)=a$ for all $a \in[0,1]$; (2) $T\left(a_{1}, \ldots, a_{n}\right)=T\left(T\left(a_{1}, \ldots, a_{n-1}\right), a_{n}\right)$ for all $n>1$. This multi-argument function $T$ is also designated t-norm. Note that $T$ is $n$-decreasing. Furthermore, $T$ defines a multi-argument function on $[0,1]^{X}$, which, since there is no risk of confusion thanks to the context, we denote by the same letter, as follows: $T: \bigcup_{n \in \mathbb{N}} \square_{n}^{X} \rightarrow[0,1]^{X}$ such that, for each $\left(\mu_{1}, \ldots, \mu_{n}\right) \in$ $\square_{n}^{X}, T\left(\mu_{1}, \ldots, \mu_{n}\right)$ denotes the element of $[0,1]^{X}$ defined, for each $x \in X$, by $T\left(\mu_{1}, \ldots, \mu_{n}\right)(x)=T\left(\mu_{1}(x), \ldots, \mu_{n}(x)\right)$.

As in the classical case, given a t-norm $T$, two fuzzy sets on $X$, or their membership functions $\mu, \sigma \in[0,1]^{X}$, are $T$-compatible if there exists $x \in X$ such that $T(\mu(x), \sigma(x)) \neq 0$ or, equivalently, if $T(\mu, \sigma) \neq \mu_{\emptyset}$. This can be similarly generalized as follows.

Definition 3.1. Given $X \neq \emptyset$ and a t-norm $T$, the set $\left\{\mu_{1}, \ldots, \mu_{n}\right\}$ $\subset[0,1]^{X}$ is said to be $T$-compatible if $T\left(\mu_{1}, \ldots, \mu_{n}\right) \neq \mu_{\emptyset}$.

Observe that $\{\mu\} \subset[0,1]^{X}$ is $T$-compatible if and only if $\mu \neq \mu_{\emptyset}$ since $T(\mu)=\mu$.

The following definition determines the conditions that a multiargument function must satisfy to fittingly assign a degree of compatibility to every $\left\{\mu_{1}, \ldots, \mu_{n}\right\} \subset[0,1]^{X}$.

Definition 3.2. Let $T$ be a t-norm and $X \neq \emptyset$. A function $\mathcal{C}: \bigcup_{n \in \mathbb{N}} \mathbb{1}_{n}^{X} \rightarrow[0,1]$ is a $T$-compatibility multi-measure on $[0,1]^{X}$ if it is a symmetric and $n$-decreasing fuzzy multi-measure on $\left([0,1]^{x}\right.$, $\left.\leqslant\right)$ satisfying $\mathcal{C}\left(\mu_{1}, \ldots, \mu_{n}\right)=0$, provided that $\left\{\mu_{1}, \ldots, \mu_{n}\right\} \subset[0,1]^{X}$ is not $T$-compatible.

The following equivalent definition lists five axioms that characterize the compatibility multi-measures. 
Definition $3.2 *$. Let $T$ be a t-norm and $X \neq \emptyset$. A function $\mathcal{C}: \bigcup_{n \in \mathbb{N}} \square_{n}^{X} \rightarrow[0,1]$ is a $T$-compatibility multi-measure on $[0,1]^{X}$ if it satisfies, for each $n \in \mathbb{N}$,

c. $1 \mathcal{C}\left(\mu_{X}, \stackrel{n}{n} ., \mu_{X}\right)=1$.

c. $2 \mathcal{C}\left(\mu_{1}, \ldots, \mu_{n}\right)=0$ for all $\left\{\mu_{1}, \ldots, \mu_{n}\right\} \subset[0,1]^{X}$ such that $T\left(\mu_{1}, \ldots, \mu_{n}\right)=\mu_{\emptyset}$.

c.3 $\mathcal{C}\left(\mu_{1}, \ldots, \mu_{n}\right)=\mathcal{C}\left(\mu_{\pi(1)}, \ldots, \mu_{\pi(n)}\right)$ holds for all $\pi \in S_{n}$ and $\mu_{1}, \ldots, \mu_{n} \in[0,1]^{X}$.

c.4 Given $\mu_{1}, \ldots, \mu_{n}, \sigma_{1}, \ldots, \sigma_{n} \in[0,1]^{X}$, if $\mu_{i} \leqslant \sigma_{i}$ holds for all $i \in\{1, \ldots, n\}$, then $\mathcal{C}\left(\mu_{1}, \ldots, \mu_{n}\right) \leqslant \mathcal{C}\left(\sigma_{1}, \ldots, \sigma_{n}\right)$.

c.5 $\mathcal{C}\left(\mu_{1}, \ldots, \mu_{n+1}\right) \leqslant \mathcal{C}\left(\mu_{1}, \ldots, \mu_{n}\right)$ holds for all $n \in \mathbb{N}$ and $\mu_{1}, \ldots, \mu_{n+1} \in[0,1]^{X}$.

If $\mathcal{C}$ satisfies axioms c.1-c.4 for some fixed $n \in \mathbb{N}$, we say that $\mathcal{C}$ is a $T$-compatibility $n$-measure on $[0,1]^{X}$. If $n=2$ we simply say that $\mathcal{C}$ is a T-compatibility measure.

Following on from Proposition 2.10, some compatibility multimeasures can be constructed as discussed below.

Proposition 3.3. Let $X \neq \emptyset$. If $T$ and $T_{0}$ are $t$-norms and $m:[0,1]^{X} \rightarrow[0,1]$ is a fuzzy measure on $\left([0,1]^{X}, \leqslant\right)$, then the functions $\mathcal{C}_{T * m}, \mathcal{C}_{m * T}: \bigcup_{n \in \mathbb{N}} \nabla_{n}^{X} \rightarrow[0,1]$, defined, for each $\left(\mu_{1}, \ldots, \mu_{n}\right) \in \square_{n}^{X}$ and $n \in \mathbb{N}$, by

$$
\begin{aligned}
& \mathcal{C}_{T * m}\left(\mu_{1}, \ldots, \mu_{n}\right)= \begin{cases}0 & \text { if } T_{0}\left(\mu_{1}, \ldots, \mu_{n}\right)=\mu_{\emptyset} \\
T\left(m\left(\mu_{1}\right), \ldots, m\left(\mu_{n}\right)\right) & \text { otherwise }\end{cases} \\
& \mathcal{C}_{m * T}\left(\mu_{1}, \ldots, \mu_{n}\right)= \begin{cases}0 & \text { if } T_{0}\left(\mu_{1}, \ldots, \mu_{n}\right)=\mu_{\emptyset} \\
m\left(T\left(\mu_{1}, \ldots, \mu_{n}\right)\right) & \text { otherwise }\end{cases}
\end{aligned}
$$

are $T_{0}$-compatibility multi-measures on $[0,1\}^{X}$.

Proof. Since the functions are defined piecewise, then the exact result cannot be obtained as a particular case of Proposition 2.10. Hence we will check directly that the axioms of compatibility measures are satisfied for the case of $\mathcal{C}_{T * m}$, the other case is similar.

(c.1) $\mathcal{C}_{T+m}\left(\mu_{X}, \ldots, \mu_{X}\right)=T\left(m\left(\mu_{X}\right), \ldots, m\left(\mu_{X}\right)\right)=T(1, \ldots, 1)=1$.

(c.2) $\mathcal{C}_{T+m}$ satisfies axiom c.2 by definition.

(c.3) As $T$ is symmetric, it follows that $\mathcal{C}_{T * m}$ is symmetric.

(c.4) If $\mu_{1}, \ldots, \mu_{n}, \sigma_{1}, \ldots, \sigma_{n} \in[0,1]^{X}$ are such that $\mu_{i} \leqslant \sigma_{i}$ for all $i \in\{1, \ldots, n\}$, we have:

- If $T_{0}\left(\sigma_{1}, \ldots, \sigma_{n}\right)=\mu_{\emptyset}$, then $T_{0}\left(\mu_{1}, \ldots, \mu_{n}\right)=\mu_{\emptyset}$, since $T_{0}$ is monotonic increasing; hence $\mathcal{C}_{T * m}\left(\mu_{1}, \ldots, \mu_{n}\right)=$ $\mathcal{C}_{T+m}\left(\sigma_{1}, \ldots, \sigma_{n}\right)=0$.

- If $T_{0}\left(\sigma_{1}, \ldots, \sigma_{n}\right) \neq \mu_{\emptyset}, \quad$ then $\quad \mathcal{C}_{T * m}\left(\mu_{1}, \ldots, \mu_{n}\right) \leqslant$ $\mathcal{C}_{T * m}\left(\sigma_{1}, \ldots, \sigma_{n}\right)$ holds either because $T$ is increasing and $m\left(\mu_{i}\right) \leqslant m\left(\sigma_{i}\right)$ for all $i \in\{1, \ldots, n\}$, or because $T_{0}\left(\mu_{1}, \ldots, \mu_{n}\right)=\mu_{\emptyset}$.

(c.5) Let $\mu_{1}, \ldots, \mu_{n}, \mu_{n+1} \in[0,1]^{X}$. If $T_{0}\left(\mu_{1}, \ldots, \mu_{n}, \mu_{n+1}\right)=\mu_{\tilde{\emptyset}}$ then $\mathcal{C}_{T * m}\left(\mu_{1}, \ldots, \mu_{n}, \mu_{n+1}\right)=0 \leqslant \mathcal{C}_{T * m}\left(\mu_{1}, \ldots, \mu_{n}\right)$; otherwise it follows that $\mathcal{C}_{T * m}\left(\mu_{1}, \ldots, \mu_{n}, \mu_{n+1}\right) \leqslant \mathcal{C}_{T+m}\left(\mu_{1}, \ldots, \mu_{n}\right)$ from $T$ being $n$-decreasing.

\section{Remark 3.4. Observe that:}

1. Even if $T_{0}=T$, for $\mathcal{C}_{T * m}$ to be a $T$-compatibility multi-measure, it does not suffice to take $\mathcal{C}_{T * m}\left(\mu_{1}, \ldots, \mu_{n}\right)=T\left(m\left(\mu_{1}\right), \ldots, m\left(\mu_{n}\right)\right)$, but it is necessary to define $\mathcal{C}_{T * m}$ as 0 on the sets that are not $T_{0}$-compatible, as is required to satisfy axiom c. 2 . Indeed, consider $T=T_{L}$ and the function $m_{\vee}:[0,1]^{X} \rightarrow[0,1]$, defined for each $\mu \in[0,1]^{X}$ by

$$
m_{\vee}(\mu)=\sup _{x \in X} \mu(x)
$$

which is a fuzzy measure on $\left([0,1]^{X}, \leqslant\right)$. If $X=[0,1]$ and $\mu_{1}$, $\mu_{2} \in[0,1]^{X}$ such that $\mu_{1}(x)=x$ and $\mu_{2}(x)=1-x$ for all $x \in[0,1]$, then $T_{L}\left(\mu_{1}, \mu_{2}\right)=\mu_{\emptyset}$. However,

$$
T_{L}\left(m_{\vee}\left(\mu_{1}\right), m_{\vee}\left(\mu_{2}\right)\right)=T_{L}(1,1)=1 .
$$

2. If $T \leqslant T_{0}$ then $\mathcal{C}_{m * T}\left(\mu_{1}, \ldots, \mu_{n}\right)=m\left(T\left(\mu_{1}, \ldots, \mu_{n}\right)\right)$ for all $\left(\mu_{1}, \ldots, \mu_{n}\right) \in \mathbb{1}_{n}^{X}$.

If we consider $m=m_{\vee}$ in the previous proposition, then we get the following result.

Corollary 3.5. Let $X \neq \emptyset$ and let $T$ and $T_{0}$ be t-norms, then the functions $\mathcal{C}_{T * V}, \mathcal{C}_{\vee * T}: \bigcup_{n \in \mathbb{N}} \square_{n}^{X} \rightarrow[0,1]$, defined for each $\left(\mu_{1}, \ldots, \mu_{n}\right) \in$ $\square_{n}^{X}$ and $n \in \mathbb{N}$, by

$$
\begin{aligned}
& \mathcal{C}_{T * \vee}\left(\mu_{1}, \ldots, \mu_{n}\right)= \begin{cases}0 & \text { if } T_{0}\left(\mu_{1}, \ldots, \mu_{n}\right)=\mu_{\emptyset} \\
T\left(\sup _{x \in X} \mu_{1}(x), \ldots, \sup _{x \in X} \mu_{n}(x)\right) & \text { otherwise }\end{cases} \\
& \mathcal{C}_{\vee * T}\left(\mu_{1}, \ldots, \mu_{n}\right)= \begin{cases}0 & \text { if } T_{0}\left(\mu_{1}, \ldots, \mu_{n}\right)=\mu_{\emptyset} \\
\sup _{x \in X} T\left(\mu_{1}(x), \ldots, \mu_{n}(x)\right) & \text { otherwise }\end{cases}
\end{aligned}
$$

are T-compatibility multi-measures on $[0,1]^{\mathrm{X}}$.

Now, let us look at some particular cases of compatibility multimeasures depending on different t-norms. To do this, recall that if $T$ is a t-norm and $\varphi \in \mathcal{A}([0,1])$, then the function $T^{\varphi}: \bigcup_{n \in \mathbb{N}}[0,1]^{n} \rightarrow$ $[0,1]$, defined for each $\left(a_{1}, \ldots, a_{n}\right) \in[0,1]^{n}$ by $T^{\varphi}\left(a_{1}, \ldots, a_{n}\right)=$ $\varphi^{-1}\left(T\left(\varphi\left(a_{1}\right), \ldots, \varphi\left(a_{n}\right)\right)\right)$, is also a t-norm. We say that $T^{\varphi}$ is the t-norm $\varphi$-conjugated with $T$. For each $\varphi \in \mathcal{A}([0,1])$ and for all $a_{1}, \ldots, a_{n} \in[0,1]$, we have that $T_{L}^{\varphi}\left(a_{1}, \ldots, a_{n}\right)=\varphi^{-1}$ $\left(\max \left\{0, \sum_{i=1}^{n} \varphi\left(a_{i}\right)-(n-1)\right\}\right), T_{P}^{\varphi}\left(a_{1}, \ldots, a_{n}\right)=\varphi^{-1}\left(\prod_{i=1}^{n} \varphi\left(a_{i}\right)\right)$ and $\operatorname{Min}^{\varphi}=$ Min. For more details about t-norms see $[1,30]$.

Corollary 3.6. Consider the t-norm $\varphi$-conjugated with $T_{L}$ and the $t$-norm $\varphi$-conjugated with $T_{P}$ for each $\varphi \in \mathcal{A}([0,1])$, and the $t$-norm Min. Given $X \neq \emptyset$,

1. Let $\mathcal{C}_{L * \vee}^{\varphi}, \mathcal{C}_{\vee * L}^{\varphi}: \bigcup_{n \in \mathbb{N}} \square_{n}^{X} \rightarrow[0,1]$ be the functions defined, for each $\left(\mu_{1}, \ldots, \mu_{n}\right) \in \mathbb{1}_{n}^{X}$ and $n \in \mathbb{N}$, by

$$
\begin{aligned}
& \mathcal{C}_{L \star \gamma}^{\varphi}\left(\mu_{1}, \ldots, \mu_{n}\right)=\left\{\begin{array}{ll}
0 & \text { if } T_{L}^{\varphi}\left(\mu_{1}, \ldots, \mu_{n}\right)=\mu_{\emptyset} \\
\max \left\{0, \sum_{i=1}^{n} \sup _{x \in X} \varphi\left(\mu_{i}(x)\right)-(n-1)\right\} & \text { otherwise }
\end{array},\right. \\
& \mathcal{C}_{\vee * L}^{\varphi}\left(\mu_{1}, \ldots, \mu_{n}\right)=\max \left\{0, \sup _{x \in X} \sum_{i=1}^{n} \varphi\left(\mu_{i}(x)\right)-(n-1)\right\} .
\end{aligned}
$$

Then, for all $\psi \in \mathcal{A}([0,1]), \psi \circ \mathcal{C}_{L * \vee}^{\varphi}$ and $\psi \circ \mathcal{C}_{\vee * L}^{\varphi}$ are $T_{L}^{\varphi}$-compatibility multi-measures on $[0,1]^{X}$ and, in particular, so are $\mathcal{C}_{L * \vee}^{\varphi}$ and $\mathcal{C}_{\vee * L}^{\varphi}$.

2. Let $\mathcal{C}_{P_{* V}}^{\varphi}, \mathcal{C}_{\vee * P}^{\varphi}: \bigcup_{n \in \mathbb{N}} \square_{n}^{X} \rightarrow[0,1]$ be the functions defined, for each $\left(\mu_{1}, \ldots, \mu_{n}\right) \in \mathbb{1}_{n}^{X}$ and $n \in \mathbb{N}$, by

$$
\begin{aligned}
& \mathcal{C}_{P * \vee}^{\varphi}\left(\mu_{1}, \ldots, \mu_{n}\right)=\left\{\begin{array}{ll}
0 & \text { if } T_{P}^{\varphi}\left(\mu_{1}, \ldots, \mu_{n}\right)=\mu_{\emptyset} \\
\prod_{i=1}^{n} \sup _{x \in X} \varphi\left(\mu_{i}(x)\right) & \text { otherwise }
\end{array},\right. \\
& \mathcal{C}_{\vee * P}^{\varphi}\left(\mu_{1}, \ldots, \mu_{n}\right)=\sup _{x \in X} \prod_{i=1}^{n} \varphi\left(\mu_{i}(x)\right) .
\end{aligned}
$$

Then, for all $\psi \in \mathcal{A}([0,1]), \psi \circ \mathcal{C}_{P * V}^{\varphi}$ and $\psi \circ \mathcal{C}_{\mathrm{V} * \mathrm{P}}^{\varphi}$ are $T_{\mathrm{P}}^{\varphi}$-compatibility multi-measures on $[0,1]^{X}$ and, in particular, so are $\mathcal{C}_{P \neq v}^{\varphi}$ and $\mathcal{C}_{V \neq P}^{\varphi}$.

3. Let $\mathcal{C}_{\mathrm{Min} * \vee}^{\varphi}, \mathcal{C}_{\vee * \mathrm{Min}}^{\varphi}: \bigcup_{n \in \mathbb{N}} \square_{n}^{X} \rightarrow[0,1]$ be the functions defined, for each $\left(\mu_{1}, \ldots, \mu_{n}\right) \in \mathbb{1}_{n}^{X}$ and $n \in \mathbb{N}$, by 


$$
\begin{aligned}
& \mathcal{C}_{\text {Min*V }}^{\varphi}\left(\mu_{1}, \ldots, \mu_{n}\right)=\operatorname{minsup}_{1 \leqslant i \leqslant n_{x \in X}} \varphi\left(\mu_{i}(x)\right), \\
& \mathcal{C}_{\vee * \operatorname{Min}}^{\varphi}\left(\mu_{1}, \ldots, \mu_{n}\right)=\sup _{x \in X} \min _{1 \leqslant i \leqslant n} \varphi\left(\mu_{i}(x)\right) .
\end{aligned}
$$

Then, for all $\psi \in \mathcal{A}([0,1]), \psi \circ \mathcal{C}_{\mathrm{Min} * \vee}^{\varphi}$ and $\psi \circ \mathcal{C}_{\mathrm{V} * \mathrm{Min}}^{\varphi}$ are Min-compatibility multi-measures on $[0,1\}^{\times}$and, in particular, so are $\mathcal{C}_{\mathrm{Min} * \vee}^{\varphi}$ and $\mathcal{C}_{\mathrm{V} * \mathrm{Min}}^{\varphi}$.

Proof. It is sufficient to check that $\mathcal{C}_{T * V}^{\varphi}=\varphi \circ \mathcal{C}_{T * V}$ and $\mathcal{C}_{\vee * T}^{\varphi}=\varphi \circ \mathcal{C}_{\vee * T}$, where $\mathcal{C}_{T * \vee}$ and $\mathcal{C}_{\vee * T}$ are the compatibility multimeasures in Corollary 3.5, in the particular cases where $T$ is $T_{L}^{\varphi}$ and $T_{P}^{\varphi}$, Min. Then, taking into account Proposition 2.9, the results are as stated above.

Remark 3.7. The function $m_{\wedge}:[0,1]^{X} \rightarrow[0,1]$, defined for each $\mu \in[0,1]^{X}$ by

$m_{\wedge}(\mu)=\inf _{x \in X} \mu(x)$,

is also a fuzzy measure. Thus, we obtain results that are analogous to those of Corollaries 3.5 and 3.6 replacing supremum with infimum in all cases. Note that, for $m_{\wedge}, \mathcal{C}_{\operatorname{Min}+\wedge}^{\varphi}=\mathcal{C}_{\wedge * \text { Min }}^{\varphi}$ holds.

Now, we introduce a new way of measuring the $T_{L}^{\varphi}$-compatibility of $\left(\mu_{1}, \ldots, \mu_{n}\right)$. Given $\left\{\mu_{1}, \ldots, \mu_{n}\right\} \subset[0,1]^{X}$, since $T_{L}^{\varphi}\left(\mu_{1}, \ldots\right.$, $\left.\mu_{n}\right) \neq \mu_{\text {ब }}$ if and only if there exists $x \in X$ such that $\sum_{i=1}^{n} \varphi\left(\mu_{i}(x)\right)>n-1$, then a good way to assign a degree of $T_{L}^{\varphi}-$ compatibility to $\left\{\mu_{1}, \ldots, \mu_{n}\right\}$ might be to fittingly take into account the difference between $\sum_{i=1}^{n} \varphi\left(\mu_{i}(x)\right) /(n-1)$ and 1 as follows.

Proposition 3.8. Given $X \neq \emptyset$ and $\varphi \in \mathcal{A}([0,1])$, let $\mathcal{C}_{L}^{\varphi}: \bigcup_{n \in \mathbb{N}}{ }_{n}^{X} \rightarrow$ $[0,1]$ be the function defined:

1. For each $\mu \in[0,1]^{X}, \mathcal{C}_{L}^{\varphi}(\mu)=\left\{\begin{array}{ll}0 & \text { if } \mu=\mu_{\emptyset} \\ 1 & \text { if } \mu \neq \mu_{\emptyset}\end{array}\right.$,

2. For each $\left(\mu_{1}, \ldots, \mu_{n}\right) \in \square_{n}^{X}$, with $n>1$,

$$
\mathcal{C}_{L}^{\varphi}\left(\mu_{1}, \ldots, \mu_{n}\right)=\left\{\begin{array}{ll}
1 & \text { if }\left(\mu_{1}, \ldots, \mu_{n}\right)=\left(\mu_{X}, \cdots, \cdot, \mu_{X}\right) \\
\max \left\{0, \sup _{X \in X} \sum_{i=1}^{n} \frac{\varphi\left(\mu_{i}(X)\right)}{n-1}-1\right\} & \text { otherwise }
\end{array} .\right.
$$

Then, for all $\psi \in \mathcal{A}([0,1]), \psi \circ \mathcal{C}_{L}^{\varphi}$ is a $T_{L}^{\varphi}$-compatibility multi-measure on $\left([0,1]^{X}, \leqslant\right)$ and, in particular, so is $\mathcal{C}_{L}^{\varphi}$.

Proof. Taking into account Proposition 2.9, it suffices to prove that $\mathcal{C}_{L}^{\varphi}$ is a $T_{L}^{\varphi}$-compatibility multi-measure on $\left([0,1]^{X}, \leqslant\right)$.

The proof of axioms c. 1 and c.3 follows directly from the definition of $\mathcal{C}_{L}^{\varphi}$. Regarding the monotony condition, given $\left(\mu_{1}, \ldots, \mu_{n}\right),\left(\sigma_{1}, \ldots, \sigma_{n}\right) \in \square_{n}^{X}$ such that $\mu_{i} \leqslant \sigma_{i}$ for all $i \in\{1, \ldots, n\}$, if $n>1, \sum_{i=1}^{n} \varphi\left(\mu_{i}(x)\right) /(n-1) \leqslant \sum_{i=1}^{n} \varphi\left(\sigma_{i}(x)\right) /(n-1)$ holds for all $x \in X$, as $\varphi$ is an increasing function; hence $\mathcal{C}_{L}^{\varphi}\left(\mu_{1}, \ldots, \mu_{n}\right) \leqslant$ $\mathcal{C}_{L}^{\varphi}\left(\sigma_{1}, \ldots, \sigma_{n}\right)$. Moreover, this inequality is satisfied trivially for the case $n=1$. Therefore, $\mathcal{C}_{l}^{\varphi}$ is a symmetric multi-measure on $\left([0,1]^{X}, \leqslant\right)$.

Furthermore, let us check that statements c.2 and c.5 are satisfied.

(c.2) If $n=1$, the axiom is satisfied by definition. If $n>1$, given $\left(\mu_{1}, \ldots, \mu_{n}\right) \in \mathbb{1}_{n}^{X}$ such that $T_{L}^{\varphi}\left(\mu_{1}, \ldots, \mu_{n}\right)=\mu_{\emptyset}$, it follows that $\sum_{i=1}^{n} \varphi\left(\mu_{i}(x)\right) \leqslant n-1$ for all $x \in X$, and so $\mathcal{C}_{L}^{\varphi}\left(\mu_{1}, \ldots, \mu_{n}\right)=0$.

(c.5) If $n=1$, let $\mu_{1} \in[0,1]^{X}$, then, for all $\mu_{2} \in[0,1]^{X}$, we have:

$\mu_{1} \neq \mu_{\emptyset} \Rightarrow \mathcal{C}_{L}^{\varphi}\left(\mu_{1}, \mu_{2}\right) \leqslant 1=\mathcal{C}_{L}^{\varphi}\left(\mu_{1}\right)$,

$\mu_{1}=\mu_{\emptyset} \Rightarrow \mathcal{C}_{L}^{\varphi}\left(\mu_{1}, \mu_{2}\right)=\max \left\{0, \sup _{x \in X} \varphi\left(\mu_{2}(x)\right)-1\right\}=0=\mathcal{C}_{L}^{\varphi}\left(\mu_{1}\right)$.

Now, let $n>1$. If $\left.\left(\mu_{1}, \ldots, \mu_{n}\right)=\left(\mu_{X}, . n\right), \mu_{X}\right)$, then
$\mathcal{C}_{L}^{\varphi}\left(\mu_{1}, \ldots, \mu_{n}\right)=1 \geqslant \mathcal{C}_{L}^{\varphi}\left(\mu_{1}, \ldots, \mu_{n+1}\right) \quad \forall \mu_{n+1} \in[0,1]^{X}$.

If $\left.\left(\mu_{1}, \ldots, \mu_{n}\right) \neq\left(\mu_{X}, . n\right), \mu_{X}\right)$, then

$$
\begin{aligned}
\mathcal{C}_{L}^{\varphi}\left(\mu_{1}, \ldots, \mu_{n}\right) & =\max \left\{0, \sup _{x \in X} \sum_{i=1}^{n} \frac{\varphi\left(\mu_{i}(x)\right)}{n-1}-1\right\} \\
& =\sup _{x \in X} \max \left\{0, \sum_{i=1}^{n} \frac{\varphi\left(\mu_{i}(x)\right)}{n-1}-1\right\} .
\end{aligned}
$$

Moreover, for all $\mu_{n+1} \in[0,1]^{X}$, also $\left(\mu_{1}, \ldots, \mu_{n}, \mu_{n+1}\right) \neq\left(\mu_{X}, \stackrel{n+1)}{+\cdots}, \mu_{X}\right)$ and then

$\mathcal{C}_{L}^{\varphi}\left(\mu_{1}, \ldots, \mu_{n}, \mu_{n+1}\right)=\sup _{x \in X} \max \left\{0, \sum_{i=1}^{n+1} \frac{\varphi\left(\mu_{i}(x)\right)}{n}-1\right\}$

For each $x \in X$, there are two possibilities:

(a) $\sum_{i=1}^{n+1} \frac{\varphi\left(\mu_{i}(x)\right)}{n} \leqslant \sum_{i=1}^{n} \frac{\varphi\left(\mu_{i}(x)\right)}{n-1}$, and then

$$
\max \left\{0, \sum_{i=1}^{n+1} \frac{\varphi\left(\mu_{i}(x)\right)}{n}-1\right\} \leqslant \max \left\{0, \sum_{i=1}^{n} \frac{\varphi\left(\mu_{i}(x)\right)}{n-1}-1\right\} .
$$

(b) $\sum_{i=1}^{n+1} \frac{\varphi\left(\mu_{i}(x)\right)}{n}>\sum_{i=1}^{n} \frac{\varphi\left(\mu_{i}(x)\right)}{n-1}$, and this inequality is equivalent to

$$
(n-1) \sum_{i=1}^{n} \varphi\left(\mu_{i}(x)\right)+(n-1) \varphi\left(\mu_{n+1}(x)\right)>n \sum_{i=1}^{n} \varphi\left(\mu_{i}(x)\right),
$$

which is equivalent to

$$
(n-1) \varphi\left(\mu_{n+1}(x)\right)>\sum_{i=1}^{n} \varphi\left(\mu_{i}(x)\right) .
$$

Then $\sum_{i=1}^{n} \varphi\left(\mu_{i}(x)\right)<n-1, \quad$ as $\quad \varphi\left(\mu_{n+1}(x)\right) \leqslant 1, \quad$ and thus $\sum_{i=1}^{n+1} \varphi\left(\mu_{i}(x)\right)<n$ also holds. Hence,

$$
\max \left\{0, \sum_{i=1}^{n+1} \frac{\varphi\left(\mu_{i}(x)\right)}{n}-1\right\}=\max \left\{0, \sum_{i=1}^{n} \frac{\varphi\left(\mu_{i}(x)\right)}{n-1}-1\right\}=0
$$

Therefore,

$\sup _{x \in X} \max \left\{0, \sum_{i=1}^{n+1} \frac{\varphi\left(\mu_{i}(x)\right)}{n}-1\right\} \leqslant \sup _{x \in X} \max \left\{0, \sum_{i=1}^{n} \frac{\varphi\left(\mu_{i}(x)\right)}{n-1}-1\right\}$

and we conclude that $\mathcal{C}_{L}^{\varphi}\left(\mu_{1}, \ldots, \mu_{n}, \mu_{n+1}\right) \leqslant \mathcal{C}_{L}^{\varphi}\left(\mu_{1}, \ldots, \mu_{n}\right)$.

\subsection{Supplementary multi-measures on fuzzy sets}

As applies in the case of compatible fuzzy sets, we need a tool to model the union of fuzzy sets in order to define supplementary fuzzy sets, and t-conorms are suitable functions for this purpose. Remember that a $t$-conorm $[1,30]$ is a binary aggregation function $S$ on the unit interval $[0,1]$, which is commutative, associative, monotone increasing with respect to the usual order on the real line, whose neutral element is 0 . The dual t-conorms of t-norms $T_{L}, T_{P}$ and Min are, respectively, the so-called Lukasiewicz, probabilistic sum and maximum t-conorms, which are defined, respectively, by $S_{L}(a, b)=\min \{1, a+b\}, S_{P}(a, b)=a+b-a \cdot b$ and $\operatorname{Max}(a, b)=\max \{a, b\}$, for each $(a, b) \in[0,1]^{2}$.

As in the t-norm case, a t-conorm $S$ also defines the symmetric fuzzy multi-measure $S: \bigcup_{n \in \mathbb{N}}[0,1]^{n} \rightarrow[0,1]$, and the multi-argument function $S: \bigcup_{n \in \mathbb{N}} \square_{n}^{X} \rightarrow[0,1]^{X}$.

Given a t-conorm $S$, two fuzzy sets on $X$ or their membership functions $\mu, \sigma \in[0,1]^{X}$ are $S$-supplementary [15] if $S(\mu(x), \sigma(x))=1$ for all $x \in X$, that is, if $S(\mu, \sigma)=\mu_{X}$. This can be generalized similarly as follows. 
Definition 3.9. Given $X \neq \emptyset$ and a t-conorm $S$, the set $\left\{\mu_{1}, \ldots, \mu_{n}\right\}$ $\subset[0,1]^{X}$ is said to be $S$-supplementary if $S\left(\mu_{1}, \ldots, \mu_{n}\right)=\mu_{X}$.

Observe that $\{\mu\} \subset[0,1]^{X}$ is $S$-supplementary if and only if $\mu=\mu_{X}$ since $S(\mu)=\mu$.

The following definition determines the conditions that a multiargument function must satisfy to fittingly assign a degree of supplementarity to every $\left\{\mu_{1}, \ldots, \mu_{n}\right\} \subset[0,1]^{X}$.

Definition 3.10. Let $S$ be a t-conorm and $X \neq \emptyset$. A function $\mathcal{S}: \cup_{n \in \mathbb{N}} \nabla_{n}^{X} \rightarrow[0,1]$ is an $S$-supplementarity multi-measure on $[0,1]^{X}$ if it is a symmetric and $n$-increasing multi-measure on $\left([0,1]^{X}, \leqslant\right)$ satisfying $\mathcal{S}\left(\mu_{1}, \ldots, \mu_{n}\right)=0$ provided that $\left\{\mu_{1}, \ldots, \mu_{n}\right\} \subset[0,1]^{X}$ is not $S$-supplementary.

The following equivalent definition lists the axioms that characterize the supplementary multi-measures.

Definition $3.10 *$. Let $S$ be a t-conorm and $X \neq \emptyset$. A function $\mathcal{S}: \cup_{n \in \mathbb{N}} \square_{n}^{X} \rightarrow[0,1]$ is an $S$-supplementarity multi-measure on $[0,1]^{X}$ if it satisfies: For each $n \in \mathbb{N}$

s.1 $\left.\mathcal{S}\left(\mu_{\mathrm{X}},{ }^{n}\right), \mu_{\mathrm{X}}\right)=1$.

s.2 $\mathcal{S}\left(\mu_{1}, \ldots, \mu_{n}\right)=0$ for all $\left\{\mu_{1}, \ldots, \mu_{n}\right\} \subset[0,1]^{X}$ such that $S\left(\mu_{1}, \ldots, \mu_{n}\right) \neq \mu_{X}$.

s.3 $\mathcal{S}\left(\mu_{1}, \ldots, \mu_{n}\right)=\mathcal{S}\left(\mu_{\pi(1)}, \ldots, \mu_{\pi(n)}\right)$ holds for all $\pi \in S_{n}$ and $\mu_{1}, \ldots, \mu_{n} \in[0,1]^{X}$.

s.4 Given $\mu_{1}, \ldots, \mu_{n}, \sigma_{1}, \ldots, \sigma_{n} \in[0,1]^{X}$, if $\mu_{i} \leqslant \sigma_{i}$ holds for all $i \in\{1, \ldots, n\}$, then $\mathcal{S}\left(\mu_{1}, \ldots, \mu_{n}\right) \leqslant \mathcal{S}\left(\sigma_{1}, \ldots, \sigma_{n}\right)$.

s.5 $\mathcal{S}\left(\mu_{1}, \ldots, \mu_{n}\right) \leqslant \mathcal{S}\left(\mu_{1}, \ldots, \mu_{n+1}\right)$ holds for all $n \in \mathbb{N}$ and $\mu_{1}, \ldots, \mu_{n+1} \in[0,1]^{X}$.

If $\mathcal{S}$ satisfies axioms s. $1-\mathrm{s} .4$ for some fixed $n \in \mathbb{N}$, we say that $\mathcal{S}$ is an $S$-supplementarity n-measure on $[0,1]^{X}$. If $n=2$ we simply say that $\mathcal{S}$ is an $S$-supplementarity measure.

The methods for obtaining compatibility measures introduced in Proposition 3.3 can be adapted to the case of supplementarity as follows. The proof runs similarly.

Proposition 3.11. Let $X \neq \emptyset$. If $S$ and $S_{0}$ are t-conorms and $m:[0,1]^{X} \rightarrow[0,1]$ is a fuzzy measure on $\left([0,1]^{X}, \leqslant\right)$, then the functions $\mathcal{S}_{S * m}, \mathcal{S}_{m * S}: \bigcup_{n \in \mathbb{N}} \rrbracket_{n}^{X} \rightarrow[0,1]$, defined for each $\left(\mu_{1}, \ldots, \mu_{n}\right) \in \mathbb{\square}_{n}^{X}$ and $n \in \mathbb{N}$ by

$$
\begin{aligned}
& \mathcal{S}_{S \times m}\left(\mu_{1}, \ldots, \mu_{n}\right)= \begin{cases}0 & \text { if } S_{0}\left(\mu_{1}, \ldots, \mu_{n}\right) \neq \mu_{X} \\
S\left(m\left(\mu_{1}\right), \ldots, m\left(\mu_{n}\right)\right) & \text { otherwise }\end{cases} \\
& \mathcal{S}_{m * S}\left(\mu_{1}, \ldots, \mu_{n}\right)= \begin{cases}0 & \text { if } S_{0}\left(\mu_{1}, \ldots, \mu_{n}\right) \neq \mu_{X}, \\
m\left(S\left(\mu_{1}, \ldots, \mu_{n}\right)\right) & \text { otherwise }\end{cases}
\end{aligned}
$$

are $S_{0}$-supplementarity multi-measures on $[0,1]^{X}$.

Remark 3.12. Observe that:

1. Even if $S_{0}=S$, for $\mathcal{S}_{S * m}$ to be an $S$-supplementary multi-measures, it is not sufficient to just define $\mathcal{S}_{S * m}\left(\mu_{1}, \ldots, \mu_{n}\right)=$ $S\left(m\left(\mu_{1}\right), \ldots, m\left(\mu_{n}\right)\right)$, but, in order to fulfill axiom s.2, it is necessary to consider $\mathcal{S}_{S * m}$ as 0 on fuzzy sets that are not $S$-supplementary. Indeed, let $X \neq \emptyset$ and $m:[0,1]^{X} \rightarrow[0,1]$ be a fuzzy measure such that $m_{\wedge} \leqslant m$; if $S=S_{L}$, and $\mu_{1}, \mu_{2} \in[0,1]^{X}$ such that $\mu_{1}=\mu_{\emptyset}$ and $\mu_{2}(x)=a$ for all $x \in X$ and $a \in(0,1)$ being a fixed value, then

$$
\begin{aligned}
& S_{L}\left(\mu_{1}, \mu_{2}\right) \neq \mu_{X}, \\
& S_{L}\left(m\left(\mu_{1}\right), m\left(\mu_{2}\right)\right)=\min \left\{1, m\left(\mu_{2}\right)\right\}=m\left(\mu_{2}\right) \geqslant a>0 .
\end{aligned}
$$

2. The multi-measure $\mathcal{S}_{S * m}$ is not worth considering when $S \geqslant S_{0}$ because it is a trivial measure. Indeed,

$$
\mathcal{S}_{m *}\left(\mu_{1}, \ldots, \mu_{n}\right)= \begin{cases}0 & \text { if } S_{0}\left(\mu_{1}, \ldots, \mu_{n}\right) \neq \mu_{x} \\ m\left(S\left(\mu_{1}, \ldots, \mu_{n}\right)\right)=m\left(\mu_{X}\right)=1 & \text { otherwise }\end{cases}
$$

As in the case of compatibility multi-measures, we mention some particular cases of supplementarity multi-measures depending on different $\mathrm{t}$-conorms. To do this, recall that if $S$ is a t-conorm and $\varphi \in \mathcal{A}([0,1])$, then $S^{\varphi}: \bigcup_{n \in \mathbb{N}}[0,1]^{n} \rightarrow[0,1]$, defined for each $\left(a_{1}, \ldots, a_{n}\right) \in[0,1]^{n}$ by $S^{\varphi}\left(a_{1}, \ldots, a_{n}\right)=\varphi^{-1}\left(S\left(\varphi\left(a_{1}\right), \ldots, \varphi\left(a_{n}\right)\right)\right)$, is also a t-conorm. We say that $S^{\varphi}$ is the t-conorm $\varphi$-conjugated with $S$. For each $\varphi \in \mathcal{A}([0,1])$ and for all $a_{1}, \ldots, a_{n} \in[0,1]$, we have that $S_{L}^{\varphi}\left(a_{1}, \ldots, a_{n}\right)=\varphi^{-1}\left(\min \left\{1, \sum_{i=1}^{n} \varphi\left(a_{i}\right)\right\}\right), S_{p}^{\varphi}\left(a_{1}, \ldots, a_{n}\right)=\varphi^{-1}\left(1-\prod_{i=1}^{n}\right.$ $\left.\left(1-\varphi\left(a_{i}\right)\right)\right)$ and $\operatorname{Max}^{\varphi}=\operatorname{Max}($ see $[1,30])$. Thus, we obtain the following result.

Corollary 3.13. Consider the t-conorm Max and, for each $\varphi \in \mathcal{A}([0,1])$, the $t$-conorm $\varphi$-conjugated with $S_{L}$ and the t-norm $\varphi$-conjugated with $S_{P}$. Given $X \neq \emptyset$, we have:

1. Let $\mathcal{S}_{L * \wedge}^{\varphi}: \bigcup_{n \in \mathbb{N}} \square_{n}^{X} \rightarrow[0,1]$ be the function defined for each $\left(\mu_{1}, \ldots, \mu_{n}\right) \in \mathbb{Q}_{n}^{X}$ and $n \in \mathbb{N}$ by

$$
\mathcal{S}_{L \star \lambda}^{\varphi}\left(\mu_{1}, \ldots, \mu_{n}\right)= \begin{cases}0 & \text { if } S_{L}^{\varphi}\left(\mu_{1}, \ldots, \mu_{n}\right) \neq \mu_{X} \\ \min \left\{1, \sum_{i=1}^{n} \inf _{x \in X} \varphi\left(\mu_{i}(x)\right)\right\} & \text { otherwise }\end{cases}
$$

Then, for each $\psi \in \mathcal{A}([0,1]), \psi \circ \mathcal{S}_{L+\wedge}^{\varphi}$ is an $S_{L}^{\varphi}$-supplementarity multimeasure on $[0,1]^{X}$ and, in particular, so is $\mathcal{S}_{L+\wedge}^{\varphi}$.

2. Let $\mathcal{S}_{P_{* \wedge}^{\varphi}}^{\varphi}: \bigcup_{n \in \mathbb{N}_{n}^{X}} \rightarrow[0,1]$ be the function defined for each $\left(\mu_{1}, \ldots, \mu_{n}\right) \in \mathbb{\mathbb { N }}_{n}^{X}$ and $n \in \mathbb{N}$ by

$$
\mathcal{S}_{P+\Lambda}^{\varphi}\left(\mu_{1}, \ldots, \mu_{n}\right)= \begin{cases}0 & \text { if } S_{P}^{\varphi}\left(\mu_{1}, \ldots, \mu_{n}\right) \neq \mu_{X} \\ 1-\prod_{i-1}^{n}\left(1-\inf _{X \in X} \varphi\left(\mu_{i}(x)\right)\right) & \text { otherwise }\end{cases}
$$

Then, for each $\psi \in \mathcal{A}([0,1]), \psi \circ \mathcal{S}_{P \neq \wedge}^{\varphi}$ is an $S_{P}^{\varphi}$-supplementarity multimeasure on $[0,1\}^{X}$ and, in particular, so is $\mathcal{S}_{P * \lambda}^{\varphi}$.

3. Let $\mathcal{S}_{\mathrm{Max} \star \Lambda}^{\varphi}: \bigcup_{n \in \mathbb{N}} \square_{n}^{X} \rightarrow[0,1]$ be the function defined for each $\left(\mu_{1}, \ldots, \mu_{n}\right) \in \mathbb{Q}_{n}^{X}$ and $n \in \mathbb{N}$ by

$$
\mathcal{S}_{\operatorname{Max} * \Lambda}^{\varphi}\left(\mu_{1}, \ldots, \mu_{n}\right)= \begin{cases}0 & \text { if } \operatorname{Max}\left(\mu_{1}, \ldots, \mu_{n}\right) \neq \mu_{X} \\ \max _{1 \leqslant i \leqslant n} \inf _{x \in X} \varphi\left(\mu_{i}(x)\right) & \text { otherwise }\end{cases}
$$

Then, for each $\psi \in \mathcal{A}([0,1]), \psi \circ \mathcal{S}_{\text {Max }}^{\varphi}$ is a Max-supplementarity multimeasure on $[0,1]^{X}$ and, in particular, so is $\mathcal{S}_{\mathrm{Max} * \wedge}^{\varphi}$.

4. Let $\mathcal{S}_{\wedge * \operatorname{Max}}, \mathcal{S}_{\vee * \mathrm{Max}}: \bigcup_{n \in \mathbb{N}} \nabla_{n}^{X} \rightarrow[0,1]$ be the functions, defined for each $\left(\mu_{1}, \ldots, \mu_{n}\right) \in \mathbb{V}_{n}^{X}$ and $n \in \mathbb{N}$ by

$$
\begin{aligned}
& \mathcal{S}_{\wedge * \operatorname{Max}}\left(\mu_{1}, \ldots, \mu_{n}\right)= \begin{cases}0 & \text { if } S\left(\mu_{1}, \ldots, \mu_{n}\right) \neq \mu_{X} \\
\inf _{x \in X} \max _{1 \leqslant i \leqslant n}\left(\mu_{i}(x)\right) & \text { otherwise }\end{cases} \\
& \mathcal{S}_{\vee * \operatorname{Max}}\left(\mu_{1}, \ldots, \mu_{n}\right)= \begin{cases}0 & \text { if } S\left(\mu_{1}, \ldots, \mu_{n}\right) \neq \mu_{X} \\
\sup _{x \in X} \max _{1 \leqslant i \leqslant n}\left(\mu_{i}(x)\right) & \text { otherwise }\end{cases}
\end{aligned}
$$

Then, for each $\psi \in \mathcal{A}([0,1]), \psi \circ \mathcal{S}_{\wedge+\mathrm{Max}}$ and $\psi \circ \mathcal{S}_{\vee+\mathrm{Max}}$ are $S$-supplementarity multi-measures on $[0,1]^{X}$ for any t-conorm $S$ and, in particular, so are $\mathcal{S}_{\wedge \neq \text { Max }}$ and $\mathcal{S}_{\vee \neq \text { Max. }}$

Remark 3.14. We can take $m=m_{\vee}$ in Proposition 3.11. Nevertheless, $\mathcal{S}_{S * \vee}$ is of no interest when the t-conorm $S$ takes the value 1 if and only if some of their arguments are 1 , as is the case of the maximum and the strict $\mathbf{t}$-conorms, that is, the $\mathbf{t}$-conorms conjugated with $S_{P}$. Indeed, in this case, $\mathcal{S}_{S * \checkmark}$ is a trivial measure since if $S\left(\mu_{1}, \ldots, \mu_{n}\right)=\mu_{X}$, then for each $x \in X$ there exists $i \in\{1, \ldots, n\}$ such that $\mu_{i}(x)=1$, and so

$$
\mathcal{S}_{S+v}\left(\mu_{1}, \ldots, \mu_{n}\right)= \begin{cases}0 & \text { if } S_{0}\left(\mu_{1}, \ldots, \mu_{n}\right) \neq \mu_{X} \\ \mathcal{S}_{S * v}\left(\mu_{1}, \ldots, \mu_{n}\right)=S\left(\sup \mu_{1}(x), \ldots, \sup \mu_{n}(x)\right)=1 & \text { otherwise }\end{cases}
$$


In the case of t-conorms conjugated with the Lukasiewicz t-conorm, there is yet another way to measure the $S_{L}^{\varphi}$-supplementarity of $\left\{\mu_{1}, \ldots, \mu_{n}\right\} \subset[0,1]^{X}$. Note that $S_{L}^{\varphi}\left(\mu_{1}, \ldots, \mu_{n}\right)=\mu_{X}$ if and only if $\sum_{i=1}^{n} \varphi\left(\mu_{i}(x)\right) \geqslant 1$ for all $x \in X$; hence we can fittingly use the difference between 1 and $\sum_{i=1}^{n} \varphi\left(\mu_{i}(x)\right)$. So, we can prove the following result.

Proposition 3.15. Given $X \neq \emptyset$ and $\varphi \in \mathcal{A}([0,1])$, let $\mathcal{S}^{\varphi}: \bigcup_{n \in \mathbb{N}} \mathbb{X}{ }_{n}^{X} \rightarrow$ $[0,1]$ be the function defined:

1. For each $\mu \in[0,1]^{X}$ by $\mathcal{S}^{\varphi}(\mu)= \begin{cases}1 & \text { if } \mu=\mu_{X} \\ 0 & \text { if } \mu \neq \mu_{X}\end{cases}$

2. For each $\left(\mu_{1}, \ldots, \mu_{n}\right) \in \square_{n}^{X}$ with $n>1$ by $\mu \neq \mu_{X}$

$$
\mathcal{S}^{\varphi}\left(\mu_{1}, \ldots, \mu_{n}\right)=\min \left\{1, \max \left\{0, \inf _{x \in X} \sum_{i=1}^{n} \varphi\left(\mu_{i}(x)\right)-1\right\}\right\} .
$$

Then, for all $\psi \in \mathcal{A}([0,1]), \psi \circ \mathcal{S}^{\varphi}$ is an $S_{L}^{\varphi}$-compatibility multi-measure on $\left([0,1]^{X}, \leqslant\right)$ and, in particular, so is $\mathcal{S}^{\varphi}$.

Remark 3.16. We have that $\mathcal{S}^{\varphi}\left(\mu_{1}, \mu_{2}\right)=\max \left\{0, \inf _{x \in X}\right.$ $\left.\left(\varphi\left(\mu_{1}(x)\right)+\varphi\left(\mu_{2}(x)\right)\right)-1\right\}$ for each $\left(\mu_{1}, \mu_{2}\right) \in \square_{2}^{X}$, and the restriction of $\mathcal{S}^{\varphi}$ to $\square_{2}^{X}=[0,1]^{X} \times[0,1]^{X}$ is just an $S_{L}^{\varphi}$-supplementarity measure regarding the definition reported in [15].

\section{Fuzzy multi-measures on lattice $\left([0,1]^{X}, \geqslant\right)$}

In this section, we deal with multi-measures on $\mathcal{L}=$ $\left([0,1]^{X}, \preceq, \mu_{\wedge}, \mu_{\vee}\right)$, where $\preceq$ is the order induced by the usual reverse order of real numbers, which is denoted in the same way. In other words, if $\mu, \sigma \in[0,1]^{X}, \mu \geqslant \sigma$ if and only if $\mu(x) \geqslant \sigma(x)$ for all $x \in X$. In this case, $\mu_{\wedge}=\mu_{X}$ and $\mu_{\vee}=\mu_{\emptyset}$, then the lattice $\mathcal{L}$ is $\left([0,1]^{X}, \geqslant, \mu_{X}, \mu_{\emptyset}\right)$, and we denote concisely $\left([0,1]^{X}, \geqslant\right)$.

Let us look at two types of multi-measures on $\left([0,1]^{x}, \geqslant\right)$. The first evaluates how incompatible a family of fuzzy sets is. The second evaluates how unsupplementary the family is, where the concepts of incompatibility and unsupplementarity are opposite to compatibility and supplementarity, respectively. In other words, given a t-norm $T$ and a t-conorm $S,\left\{\mu_{1}, \ldots, \mu_{n}\right\} \subset \square_{n}^{X}$ is T-incompatible if it is not T-compatible, and it is $S$-unsupplementary if it is not $S$-supplementary.

\subsection{Incompatibility multi-measures on fuzzy sets}

Although compatibility and incompatibility are opposites concepts, the negation of a compatibility measure cannot be used to assign degrees of incompatibility. Indeed, suppose that $\mathcal{C}$ is a nontrivial $T$-compatibility multi-measure, that is, it takes one value $a \neq 0,1$, and consider a strong negation $N[51]$ (i.e. $N:[0,1] \rightarrow[0,1]$ is an involutive and decreasing bijection). If $a$ is achieved by $\mathcal{C}$ on $\left(\mu_{1}, \ldots \mu_{n}\right) \in \mathbb{n}_{n}^{X}$, then $0<\mathcal{C}\left(\mu_{1}, \ldots \mu_{n}\right)=a<1$, and, on the one hand, it follows from axiom c. 2 of Definition $3.2 *$ that $\left\{\mu_{1}, \ldots, \mu_{n}\right\}$ is $T$-compatible, and on the other hand, $0=N(1)<N\left(\mathcal{C}\left(\mu_{1}, \ldots \mu_{n}\right)\right)<$ $N(0)=1$ holds. Thus $N\left(\mathcal{C}\left(\mu_{1}, \ldots \mu_{n}\right)\right)$ cannot be considered as a degree of the $T$-incompatibility of $\left\{\mu_{1}, \ldots, \mu_{n}\right\}$ since the incompatibility measure of compatible sets should be 0 . Therefore, it makes sense to propose a mathematical model for study incompatibility.

Given a t-norm $T$, we have that $\left\{\mu_{1}, \ldots, \mu_{n}\right\} \subset[0,1]^{X}$ is T-incompatible if it is not T-compatible, that is, if $T\left(\mu_{1}, \ldots, \mu_{n}\right)=\mu_{\emptyset}$. The following definition determines the requirements that a function should satisfy to be considered an incompatibility multi-measure.

Definition 4.1. Let $T$ be a t-norm and $X \neq \emptyset$. A function $\mathcal{I}: \bigcup_{n \in \mathbb{N}} \mathbb{N}_{n}^{X} \rightarrow[0,1]$ is a T-incompatibility multi-measure on $[0,1]^{X}$ if it is a symmetric and $n$-increasing multi-measure on $\left([0,1]^{X}, \geqslant\right)$ satisfying $\mathcal{I}\left(\mu_{1}, \ldots, \mu_{n}\right)=0$, provided that $T\left(\mu_{1}, \ldots, \mu_{n}\right) \neq \mu_{\emptyset}$.
Now, we list the five axioms of an incompatibility multimeasure

Definition 4.1 $*$. Let $T$ be a t-norm and $X \neq \emptyset$. A function $\mathcal{I}: \bigcup_{n \in \mathbb{N}} \square_{n}^{X} \rightarrow[0,1]$ is a $T$-incompatibility multi-measure on $[0,1]^{X}$ if it satisfies, for each $n \in \mathbb{N}$,

i.1 $\mathcal{I}\left(\mu_{\tilde{\natural}}, \cdots, ., \mu_{\emptyset}\right)=1$.

i.2 $\mathcal{I}\left(\mu_{1}, \ldots, \mu_{n}\right)=0$ for all $\left\{\mu_{1}, \ldots, \mu_{n}\right\} \subset[0,1]^{X}$ such that $T\left(\mu_{1}, \ldots, \mu_{n}\right) \neq \mu_{\emptyset}$.

i.3 $\mathcal{I}\left(\mu_{1}, \ldots, \mu_{n}\right)=\mathcal{I}\left(\mu_{\pi(1)}, \ldots, \mu_{\pi(n)}\right)$ holds for all $\pi \in S_{n}$ and $\mu_{1}, \ldots, \mu_{n} \in[0,1]^{X}$.

i.4 Given $\mu_{1}, \ldots, \mu_{n}, \sigma_{1}, \ldots, \sigma_{n} \in[0,1]^{X}$, if $\mu_{i} \leqslant \sigma_{i}$ holds for all $i \in\{1, \ldots, n\}$, then $\mathcal{I}\left(\sigma_{1}, \ldots, \sigma_{n}\right) \leqslant \mathcal{I}\left(\mu_{1}, \ldots, \mu_{n}\right)$.

i.5 $\mathcal{I}\left(\mu_{1}, \ldots, \mu_{n}\right) \leqslant \mathcal{I}\left(\mu_{1}, \ldots, \mu_{n+1}\right)$ holds for all $n \in \mathbb{N}$ and $\mu_{1}, \ldots, \mu_{n+1} \in[0,1]^{X}$.

If $\mathcal{I}$ satisfies axioms i.1-i.4 for some fixed $n \in \mathbb{N}$, we say that $\mathcal{I}$ is a $T$-incompatibility n-measure on $[0,1]^{X}$. If $n=2$, we simply say that $\mathcal{I}$ is a $T$-incompatibility measure.

Again, Proposition 2.10 provides a method to obtain incompatibility multi-measures. But now the lattice to be considered is $\mathcal{L}=\left([0,1]^{X}, \geqslant, \mu_{X}, \mu_{0}\right)$, and we need a fuzzy measure $m^{\prime}:[0,1]^{X} \rightarrow$ $[0,1]$ on $\mathcal{L}$. Thus, $m^{\prime}$ must satisfy:

1. $m^{\prime}\left(\mu_{X}\right)=0$ and $m^{\prime}\left(\mu_{\emptyset}\right)=1$.

2. Given $\mu, \sigma \in[0,1]^{X}$, if $\mu \geqslant \sigma$ then $m^{\prime}(\mu) \leqslant m^{\prime}(\sigma)$.

Proposition 4.2. If $T$ and $T_{0}$ are t-norms, $S$ is a $t$-conorm and $m^{\prime}:[0,1]^{X} \rightarrow[0,1]$ is a fuzzy measure on $\left([0,1]^{X}, \geqslant\right)$, where $X \neq \emptyset$, then the functions $\mathcal{I}_{S * m^{\prime}}, \mathcal{I}_{m^{\prime} * S}: \bigcup_{n \in \mathbb{N}} \square_{n}^{X} \rightarrow[0,1]$, defined for each $\left(\mu_{1}, \ldots, \mu_{n}\right) \in \mathbb{R}_{n}^{X}$ and $n \in \mathbb{N}$ by

$$
\begin{aligned}
& \mathcal{I}_{S * m^{\prime}}\left(\mu_{1}, \ldots, \mu_{n}\right)= \begin{cases}0 & \text { if } T_{0}\left(\mu_{1}, \ldots, \mu_{n}\right) \neq \mu_{\emptyset} \\
S\left(m^{\prime}\left(\mu_{1}\right), \ldots, m^{\prime}\left(\mu_{n}\right)\right) & \text { otherwise }\end{cases} \\
& \mathcal{I}_{m^{\prime} \times T}\left(\mu_{1}, \ldots, \mu_{n}\right)= \begin{cases}0 & \text { if } T_{0}\left(\mu_{1}, \ldots, \mu_{n}\right) \neq \mu_{\emptyset} \\
m^{\prime}\left(T\left(\mu_{1}, \ldots, \mu_{n}\right)\right) & \text { otherwise }\end{cases}
\end{aligned}
$$

are $T_{0}$-incompatibility multi-measures on $[0,1]^{X}$.

Proof. As in Proposition 3.3, the proof of i.1-i.3 is straightforward Let us check the other axioms for $\mathcal{I}_{S * m^{\prime}}$, the other case is similar.

(i.4) Given $\mu_{1}, \ldots, \mu_{n}, \sigma_{1}, \ldots, \sigma_{n} \in[0,1]^{X}$ such that $\mu_{i} \leqslant \sigma_{i}$ for all $i \in\{1, \ldots, n\}$, we have:

- If $T_{0}\left(\sigma_{1}, \ldots, \sigma_{n}\right) \neq \mu_{\emptyset}$, then $\mathcal{I}_{S * m^{\prime}}\left(\sigma_{1}, \ldots, \sigma_{n}\right)=0 \leqslant$ $\mathcal{I}_{S+m^{\prime}}\left(\mu_{1}, \ldots, \mu_{n}\right)$.

- If $T_{0}\left(\sigma_{1}, \ldots, \sigma_{n}\right)=\mu_{\emptyset}$, then $T_{0}\left(\mu_{1}, \ldots, \mu_{n}\right)=\mu_{\emptyset}$, because $T_{0}$ is monotonic increasing; besides, since $S$ is increasing and $m^{\prime}\left(\sigma_{i}\right) \leqslant m^{\prime}\left(\mu_{i}\right)$ for all $i \in\{1, \ldots, n\}$, it follows that $\mathcal{I}_{S * m^{\prime}}\left(\sigma_{1}, \ldots, \sigma_{n}\right)=S\left(m^{\prime}\left(\sigma_{1}\right), \ldots, m^{\prime}\left(\sigma_{n}\right)\right) \leqslant S\left(m^{\prime}\left(\mu_{1}\right), \ldots, m^{\prime}\right.$ $\left.\left(\mu_{n}\right)\right)=\mathcal{I}_{S_{*} m^{\prime}}\left(\mu_{1}, \ldots, \mu_{n}\right)$.

(i.5) Let $\mu_{1}, \ldots, \mu_{n}, \mu_{n+1} \in[0,1]^{X}$. If $T_{0}\left(\mu_{1}, \ldots, \mu_{n}, \mu_{n+1}\right) \neq \mu_{\emptyset}$, then $T_{0}\left(\mu_{1}, \ldots, \mu_{n}\right) \neq \mu_{\emptyset}$ and $\mathcal{I}_{S * m^{\prime}}\left(\mu_{1}, \ldots, \mu_{n}, \mu_{n+1}\right)=0=$ $\mathcal{I}_{S_{*} m^{\prime}}\left(\mu_{1}, \ldots, \mu_{n}\right)$; otherwise, it follows from $S$ being $n$-increasing that $\mathcal{I}_{S * m^{\prime}}\left(\mu_{1}, \ldots, \mu_{n}, \mu_{n+1}\right) \geqslant \mathcal{I}_{S * m^{\prime}}\left(\mu_{1}, \ldots, \mu_{n}\right)$.

Remark 4.3. Observe that:

1. It does not make sense to replace the t-conorm $S$ with a t-norm $T$ in the definition of $\mathcal{I}_{S * m^{\prime}}$, because $T\left(m^{\prime}\left(\mu_{1}\right), \ldots, m^{\prime}\left(\mu_{n}\right)\right)$ $\geqslant T\left(m^{\prime}\left(\mu_{1}\right), \ldots, m^{\prime}\left(\mu_{n}\right), m^{\prime}\left(\mu_{n+1}\right)\right)$, and so axiom i.5 is not satisfied. 
2. As $S$ is $n$-increasing and $m^{\prime}$ reverses the order, then $m^{\prime} \circ S$ is $n$ decreasing. Thus, it is meaningless to replace the $t$-norm $T$ with a t-conorm $S$ in the definition of $\mathcal{I}_{m^{\prime} * T}$.

3. If $T \leqslant T_{0}$, the second formula of Proposition 4.2 produces the trivial multi-measure

$$
\mathcal{I}_{m^{\prime}+\mathrm{T}}\left(\mu_{1}, \ldots, \mu_{n}\right)= \begin{cases}0 & \text { if } T_{0}\left(\mu_{1}, \ldots, \mu_{n}\right) \neq \mu_{\emptyset} \\ m^{\prime}\left(T\left(\mu_{1}, \ldots, \mu_{n}\right)\right)=1 & \text { otherwise }\end{cases}
$$

If $m$ is a fuzzy measure on $\left([0,1]^{X}, \leqslant\right)$ and $N:[0,1] \rightarrow[0,1]$ is a negation, then $m_{N}^{\prime}=N \circ m$ is a fuzzy measure on $\left([0,1]^{X}, \geqslant\right)$. The following result explains some possible incompatibility multimeasures, as special cases of Proposition 4.2, considering $m_{\checkmark}^{\prime}=N_{s} \circ m_{\vee}$, where $N_{s}$ is the standard negation (i.e. $N_{s}(a)=1-a$ for each $a \in[0,1])$. Obviously, $m_{\wedge}^{\prime}=N_{S} \circ m_{\wedge}$ can also be used in a similar way.

Corollary 4.4. Let $X \neq \emptyset$ and $\varphi \in \mathcal{A}([0,1])$. Consider the t-norms Min and the $\varphi$-conjugated with $T_{L}$ and $T_{P}$. We have:

1. If $\mathcal{I}_{L * V^{v}}^{\varphi}: \bigcup_{n \in \mathbb{N}} \square_{n}^{X} \rightarrow[0,1]$ is the function defined for each $\left(\mu_{1}, \ldots, \mu_{n}\right) \in \mathbb{1}_{n}^{X}$ and $n \in \mathbb{N}$ by

$$
I_{L * *^{\prime}}^{\varphi}\left(\mu_{1}, \ldots, \mu_{n}\right)= \begin{cases}0 & \text { if } T_{L}^{\varphi}\left(\mu_{1}, \ldots, \mu_{n}\right) \neq \mu_{\emptyset} \\ \min \left\{1, \sum_{i=1}^{n}\left(1-\sup _{x \in X} \varphi\left(\mu_{i}(x)\right)\right)\right\} & \text { otherwise }\end{cases}
$$

then, for each $\psi \in \mathcal{A}([0,1]), \psi \circ \mathcal{I}_{L+v^{\prime}}^{\varphi}$ is a $T_{I}^{\varphi}$-incompatibility multimeasure on $[0,1\}^{X}$ and, in particular, so is $\mathcal{I}_{L \neq \nu^{\prime}}^{\varphi^{\perp}}$.

2. If $\mathcal{I}_{P_{*} \mathrm{~V}^{\prime}}^{\varphi}: \bigcup_{n \in \mathbb{N}_{n}} \nabla_{n}^{X} \rightarrow[0,1]$ is the function defined for each $\left(\mu_{1}, \ldots, \mu_{n}\right) \in \mathbb{1}_{n}^{X}$ and $n \in \mathbb{N}$ by

$$
\mathcal{I}_{P_{*} \mathcal{V}^{\prime}}^{\varphi}\left(\mu_{1}, \ldots, \mu_{n}\right)= \begin{cases}0 & \text { if } T_{P}^{\varphi}\left(\mu_{1}, \ldots, \mu_{n}\right) \neq \mu_{\emptyset} \\ 1-\prod_{i=1}^{n} \sup _{x \in X} \varphi\left(\mu_{i}(x)\right) & \text { otherwise }\end{cases}
$$

then, for each $\psi \in \mathcal{A}([0,1]), \psi \circ \mathcal{I}_{P * \vee^{\prime}}^{\varphi}$ is a $T_{P}^{\varphi}$-incompatibility multimeasure on $[0,1\}^{X}$ and, in particular, so is $\mathcal{I}_{P \neq v}^{\varphi}$.

3. If $\mathcal{I}_{\mathrm{Max} * \vee^{\prime}}^{\varphi}: \bigcup_{n \in \mathbb{N}} \mathbb{X}_{n}^{X} \rightarrow[0,1]$ is the function defined for each $\left(\mu_{1}, \ldots, \mu_{n}\right) \in \mathbb{V}_{n}^{X}$ and $n \in \mathbb{N}$ by

$$
\mathcal{I}_{\text {Max } v^{\prime}}^{\varphi}\left(\mu_{1}, \ldots, \mu_{n}\right)=\left\{\begin{array}{ll}
0 & \text { if } \operatorname{Min}\left(\mu_{1}, \ldots, \mu_{n}\right) \neq \mu_{0} \\
\max _{1 \leqslant i \leqslant n}\left(1-\sup _{x \in \mathbb{X}} \varphi\left(\mu_{i}(x)\right)\right) & \text { otherwise }
\end{array},\right.
$$

then, for each $\psi \in \mathcal{A}([0,1]), \psi \circ \mathcal{I}_{\mathrm{Max}+v^{\prime}}^{\varphi}$ is a Min-incompatibility multimeasure on $[0,1\}^{X}$ and, in particular, so is $\mathcal{I}_{\text {Max* }}^{\varphi}$.

4. If $\mathcal{I}_{\mho^{\prime}+\operatorname{Min}}: \bigcup_{n \in \mathbb{N}} \square_{n}^{X} \rightarrow[0,1]$ is the function defined for each $\left(\mu_{1}, \ldots, \mu_{n}\right) \in \mathbb{D}_{n}^{X}$ and $n \in \mathbb{N}$ by

$$
\mathcal{I}_{\mathrm{V}^{\prime} * \operatorname{Min}}\left(\mu_{1}, \ldots, \mu_{n}\right)= \begin{cases}0 & \text { if } T\left(\mu_{1}, \ldots, \mu_{n}\right) \neq \mu_{\emptyset} \\ 1-\sup _{x \in X} \min _{1 \leqslant i \leqslant n} \mu_{i}(x) & \text { otherwise }\end{cases}
$$

then, for each $\psi \in \mathcal{A}([0,1]), \psi \circ \mathcal{I}_{v^{*}+\operatorname{Min}}$ is a T-incompatibility multimeasure on $[0,1\}^{X}$ for any t-norm $T$ and, in particular, so is $\mathcal{I}_{v^{\prime}+\text { Min. }}$.

As in the case of compatibility, if $T_{L}^{\varphi}$ is the t-norm $\varphi$-conjugated with the Lukasiewicz t-norm, taking into account that $T_{L}^{\varphi}\left(\mu_{1}, \ldots, \mu_{n}\right)=\mu_{\emptyset}$ if and only if $\sum_{i=1}^{n} \varphi\left(\mu_{i}(x)\right) \leqslant n-1$, for all $x \in X$, we can find a $T_{L}^{\varphi}$-incompatibility multi-measure by fittingly considering the difference between $n-1$ and $\sum_{i=1}^{n} \varphi\left(\mu_{i}(x)\right)$, and thus we can prove the following result.

Proposition 4.5. Let $X \neq \emptyset$ and $\varphi \in \mathcal{A}([0,1])$. If $\mathcal{I}^{\varphi}: \cup_{n \in \mathbb{N}} \square_{n}^{X} \rightarrow[0,1]$ is the function defined:

1. For each $\mu \in[0,1]^{\mathrm{X}}$, by $\mathcal{I}^{\varphi}(\mu)= \begin{cases}0 & \text { if } \mu \neq \mu_{\emptyset} \\ 1 & \text { if } \mu=\mu_{\emptyset}\end{cases}$
2. For each $\left(\mu_{1}, \ldots, \mu_{n}\right) \in \mathbb{Q}_{n}^{X}$ with $n>1$, by

$$
\mathcal{I}^{\varphi}\left(\mu_{1}, \ldots, \mu_{n}\right)=\min \left\{1, \max \left\{0,(n-1)-\sup _{x \in X} \sum_{i=1}^{n} \varphi\left(\mu_{i}(x)\right)\right\}\right\},
$$

then $\mathcal{I}^{\varphi}$ is a $T_{L}^{\varphi}$-incompatibility multi-measure on $[0,1]^{X}$.

Remark 4.6. We have that $\mathcal{I}^{\varphi}\left(\mu_{1}, \mu_{2}\right)=\max \{0,1-$ $\left.\sup _{x \in X}\left(\varphi\left(\mu_{1}(x)\right)+\varphi\left(\mu_{2}(x)\right)\right)\right\}$ for each $\left.\left(\mu_{1}, \mu_{2}\right) \in\right]_{2}^{X}$, thus $\left.\mathcal{I}^{\varphi}\right|_{[0,1]^{X} \times[0,1]^{X}}$ is a $T_{L}^{\varphi}$-incompatibility measure regarding the definition reported in [11].

Lemma 4.7. Let $X \neq \emptyset$ and $\varphi \in \mathcal{A}([0,1])$. The function $I_{n}^{\varphi}: \mathbb{1}_{n}^{X} \rightarrow[0,1]$, with $n \in \mathbb{N} \backslash\{1\}$, defined for any $\left(\mu_{1}, \ldots, \mu_{n}\right) \in \mathbb{\square}_{n}^{X}$ by

$I_{n}^{\varphi}\left(\mu_{1}, \ldots, \mu_{n}\right)=\max \left\{0,1-\sup _{x \in X}\left\{\sum_{i=1}^{n} \frac{\varphi\left(\mu_{i}(x)\right)}{n-1}\right\}\right\}$

is a $T_{L}^{\varphi}$-incompatibility n-measure.

Proof. Axioms i.1 and i.3 can be proven by a simple computation.

(i.2) If $\left\{\mu_{1}, \ldots, \mu_{n}\right\} \subset[0,1]^{X}$ satisfies $T_{L}^{\varphi}\left(\mu_{1}, \ldots, \mu_{n}\right) \neq \mu_{\emptyset}$, then there exists $x \in X$ such that $\sum_{i=1}^{n} \varphi\left(\mu_{i}(x)\right)>n-1$, thus, and taking into account that $n>1$, it follows that

$$
\sup _{x \in X} \frac{\sum_{i=1}^{n} \varphi\left(\mu_{i}(x)\right)}{n-1}>1 \text {. }
$$

Therefore, $I_{n}^{\varphi}\left(\mu_{1}, \ldots, \mu_{n}\right)=0$.

(i.4) If $\mu_{1}, \ldots, \mu_{n}, \sigma_{1}, \ldots, \sigma_{n} \in[0,1]^{X}$ satisfy $\mu_{i} \leqslant \sigma_{i}$ for all $i \in\{1, \ldots, n\}$, as $\varphi$ is increasing and $n>1$, then

$$
\begin{aligned}
\forall x & \in X, \quad \frac{\sum_{i=1}^{n} \varphi\left(\mu_{i}(x)\right)}{n-1} \leqslant \frac{\sum_{i=1}^{n} \varphi\left(\sigma_{i}(x)\right)}{n-1} \\
& \Rightarrow 1-\sup _{x \in X} \frac{\sum_{i=1}^{n} \varphi\left(\sigma_{i}(x)\right)}{n-1} \leqslant 1-\sup _{x \in X} \frac{\sum_{i=1}^{n} \varphi\left(\mu_{i}(x)\right)}{n-1} .
\end{aligned}
$$

Hence, $I_{n}^{\varphi}\left(\sigma_{1}, \ldots, \sigma_{n}\right) \leqslant I_{n}^{\varphi}\left(\mu_{1}, \ldots, \mu_{n}\right)$

Remark 4.8. The above formula cannot be naturally extended to obtain a multi-measure. More precisely, there is no $T_{L}^{\varphi}$-incompatibility multi-measure $\mathcal{I}: \bigcup_{n \in \mathbb{N}} \|_{n}^{X} \rightarrow[0,1]$ such that $\left.\mathcal{I}\right|_{0_{n}^{X}}=I_{n}^{\varphi}$ for each $n>1$. Let us suppose that there exists such a multi-argument function $\mathcal{I}$, then we will deduce a contradiction. Consider $\left(\mu_{1}, \mu_{2}\right) \in \square_{2}^{X}$ such that $\mu_{1}(x)=\mu_{2}(x)=0.4$ for all $x \in X$, then $\mathcal{I}\left(\mu_{1}, \mu_{2}\right)=$ $I_{2}^{\varphi}\left(\mu_{1}, \mu_{2}\right)=\max \{0,1-(0.4+0.4)\}=0.2$. Now, let $\mu_{3}=\mu_{X}$, then $\mathcal{I}\left(\mu_{1}, \mu_{2}, \mu_{3}\right)=I_{3}^{\varphi}\left(\mu_{1}, \mu_{2}, \mu_{3}\right)=\max \left\{0,1-\frac{0.4+0.4+1}{2}\right\}=0.1$. Therefore $\mathcal{I}\left(\mu_{1}, \mu_{2}, \mu_{3}\right)=0.1<\mathcal{I}\left(\mu_{1}, \mu_{2}\right)=0.2$, and this contradicts axiom i.5 of incompatibility multi-measures. However, we can construct a multi-argument function, which is defined on each element of $\bigcup_{n \in \mathbb{N}} \square_{n}^{X}$ by means of some function $I_{n}^{\varphi}$, as it is shown in the next result.

Proposition 4.9. Given $\varphi \in \mathcal{A}([0,1])$, let $T_{I}^{\varphi}$ be the t-norm $\varphi$-conjugated with the Lukasiewicz t-norm and let $I_{n}^{\varphi}$ be the n-measure on $[0,1]^{X}$, with $X \neq \emptyset$, defined in Lemma 4.7. Consider the function $\mathcal{I}_{L}^{\varphi}: \bigcup_{n \in \mathbb{N}} \mathbb{N}_{n}^{X} \rightarrow[0,1]$ defined recursively as follows:

1. If $n=1$ : for each

$$
\mu \in[0,1]^{X}, \mathcal{I}_{L}^{\varphi}(\mu)=\left\{\begin{array}{ll}
0 & \text { if } \mu \neq \mu_{\emptyset} \\
1 & \text { if } \mu=\mu_{\emptyset}
\end{array} .\right.
$$

2. If $n>1$ : for each $\left(\mu_{1}, \ldots, \mu_{n}\right) \in \mathbb{1}_{n}^{X}$ 


$$
\mathcal{I}_{L}^{\varphi}\left(\mu_{1}, \ldots, \mu_{n}\right)=\max \left\{\max _{\left\{i_{1}, \ldots, i_{n}\right) \in \mathcal{P}\left(N_{n}\right)} \mathcal{I}_{L}^{\varphi}\left(\mu_{i_{1}}, \ldots \mu_{i_{n}}\right), I_{n}^{\varphi}\left(\mu_{1}, \ldots, \mu_{n}\right)\right\},
$$

where $\mathcal{P}\left(\mathcal{N}_{n}\right)$ denotes the power set of $\mathcal{N}_{n}=\{1,2, \ldots, n\}$.

Then, for all $\psi \in \mathcal{A}([0,1]), \psi \circ \mathcal{I}_{L}^{\varphi}$ is a $T_{L}^{\varphi}$-incompatibility multi-measure on $\left([0,1]^{X}, \leqslant\right)$ and, in particular, so is $\mathcal{I}_{L}^{\varphi}$.

Proof. Taking into account Proposition 2.9, it suffices to prove that $\mathcal{I}_{L}^{\varphi}$ is a $T_{L}^{\varphi}$-incompatibility multi-measure on $\left([0,1]^{X}, \leqslant\right)$. The proof of axioms i.1, i.3 and i.5 follows directly from the definition of $\mathcal{I}_{L}^{\varphi}$. To prove the others, we need to use induction.

(i.2) We have to prove that the statement "If $\mu_{1}, \ldots, \mu_{n} \in[0,1]^{X}$ satisfy $T_{L}^{\varphi}\left(\mu_{1}, \ldots, \mu_{n}\right) \neq \mu_{\emptyset}$, then $\mathcal{I}_{L}^{\varphi}\left(\mu_{1}, \ldots, \mu_{n}\right)=0$ " is true for all $n \in \mathbb{N}$.

Case $n=1$ : If $\mu \in[0,1]^{X}$ satisfies $T_{L}^{\varphi}(\mu) \neq \mu_{\emptyset}$, then $\mathcal{I}_{L}^{\varphi}(\mu)=0$ by definition.

Case $n>1$ : Suppose that the statement is true for $n-1$, then we will prove it for $n$. Let $\mu_{1}, \ldots, \mu_{n} \in[0,1]^{X}$ such that $T_{L}^{\varphi}\left(\mu_{1}, \ldots, \mu_{n}\right) \neq \mu_{\emptyset}$, we deduce:

- $T_{L}^{\varphi}\left(\mu_{i_{1}}, \ldots, \mu_{i_{n 1}}\right) \neq \mu_{\emptyset}$ for all $\left\{i_{1}, \ldots, i_{n-1}\right\} \in \mathcal{P}\left(\mathcal{N}_{n}\right)$, then $\mathcal{I}_{L}^{\varphi}\left(\mu_{i_{1}}, \ldots, \mu_{i_{n} 1}\right)=0$ by induction hypothesis.

- $I_{n}^{\varphi}\left(\mu_{1}, \ldots, \mu_{n}\right)=0$ holds by Lemma 4.7.

Hence, $\mathcal{I}_{L}^{\varphi}\left(\mu_{1}, \ldots, \mu_{n}\right)=0$.

Consequently, from the induction Principle we conclude that $\mathcal{I}_{L}^{\varphi}$ satisfies axiom i.2.

(i.4) We must prove that the statement "If $\mu_{1}, \ldots, \mu_{n}, \sigma_{1}, \ldots, \sigma_{n} \in$ $[0,1]^{X}$ satisfy $\mu_{i} \leqslant \sigma_{i}$ for all $i \in\{1, \ldots, n\}$, then $\mathcal{I}_{L}^{\varphi}\left(\sigma_{1}, \ldots, \sigma_{n}\right) \leqslant \mathcal{I}_{L}^{\varphi}\left(\mu_{1}, \ldots, \mu_{n}\right)$ "' is true for all $n \in \mathbb{N}$.

Case $n=1$ : Let $\mu, \sigma \in[0,1]^{X}$ such that $\mu \leqslant \sigma$. We have two cases:

- If $\mu \neq \mu_{\emptyset}$, then $\sigma \neq \mu_{\emptyset}$; therefore $\mathcal{I}_{L}^{\varphi}(\sigma)=\mathcal{I}_{L}^{\varphi}(\mu)=0$.

- If $\mu=\mu_{\emptyset}$, then $\mathcal{I}_{L}^{\varphi}(\mu)=1 \geqslant \mathcal{I}_{L}^{\varphi}(\sigma)$.

Hence, $\mathcal{I}_{L}^{\varphi}(\sigma) \leqslant \mathcal{I}_{L}^{\varphi}(\mu)$.

Case $n>1$ : Assume that the statement is true for $n-1$, then we will prove it for $n$. Let $\mu_{1}, \ldots, \mu_{n}, \sigma_{1}, \ldots, \sigma_{n} \in[0,1]^{X}$ such that $\mu_{i} \leqslant \sigma_{i}$ for all $i \in\{1, \ldots, n\}$, we deduce:

- The induction hypothesis assures that $\mathcal{I}_{L}^{\varphi}\left(\sigma_{i_{1}}, \ldots, \sigma_{i_{n 1}}\right) \leqslant$ $\mathcal{I}_{L}^{\varphi}\left(\mu_{i_{1}}, \ldots, \mu_{i_{n 1}}\right)$ holds for any $\left\{i_{1}, \ldots, i_{n-1}\right\} \in \mathcal{P}\left(\mathcal{N}_{n}\right)$, and thus

$$
\max _{\left\{i_{1}, \ldots, i_{n-1}\right\} \in \mathcal{P}\left(\mathcal{N}_{n}\right)} \mathcal{I}_{L}^{\varphi}\left(\sigma_{i_{1}}, \ldots \sigma_{i_{n-1}}\right) \leqslant \max _{\left\{i_{1}, \ldots, i_{n-1}\right\} \in \mathcal{P}\left(\mathcal{N}_{n}\right)} \mathcal{I}_{L}^{\varphi}\left(\mu_{i_{1}}, \ldots \mu_{i_{n-1}}\right) .
$$

- $I_{n}^{\varphi}\left(\sigma_{1}, \ldots, \sigma_{n}\right) \leqslant I_{n}^{\varphi}\left(\mu_{1}, \ldots, \mu_{n}\right)$ holds by Lemma 4.7 .

Hence, $\mathcal{I}_{L}^{\varphi}\left(\sigma_{1}, \ldots, \sigma_{n}\right) \leqslant \mathcal{I}_{L}^{\varphi}\left(\mu_{1}, \ldots, \mu_{n}\right)$.

Therefore, we can claim that $\mathcal{I}_{L}^{\varphi}$ satisfies axiom i.5. by the induction Principle.

\subsection{Unsupplementarity multi-measures on fuzzy sets}

As for incompatibility, although unsupplementary is the opposite to supplementary, it is not possible to assign degrees of unsupplementarity by means of a negation of a supplementarity multimeasure. Hence we establish a mathematical model to measure the unsupplementarity property.

Given a t-conorm $S$, we have that $\left\{\mu_{1}, \ldots, \mu_{n}\right\} \subset[0,1]^{X}$ is $S$-unsupplementary if it is not $S$-supplementary, that is, if $S\left(\mu_{1}\right.$, $\left.\ldots, \mu_{n}\right) \neq \mu_{\mathrm{X}}$. The following definition determines the conditions that a multi-argument function must satisfy to fittingly assign a degree of unsupplementarity to every $\left\{\mu_{1}, \ldots, \mu_{n}\right\} \subset[0,1]^{X}$.
Definition 4.10. Let $S$ be a t-conorm and $X \neq \emptyset$. A function $\mathcal{U}: \cup_{n \in \mathbb{N}} \square_{n} \rightarrow[0,1]$ is an S-unsupplementarity multi-measure on $[0,1]^{X}$ if it is a symmetric and $n$-decreasing multi-measure on $\left([0,1]^{X}, \geqslant\right)$ satisfying $\mathcal{U}\left(\mu_{1}, \ldots, \mu_{n}\right)=0$, provided that $\left\{\mu_{1}, \ldots, \mu_{n}\right\}$ $\subset[0,1]^{X}$ is $S\left(\mu_{1}, \ldots, \mu_{n}\right)=\mu_{X}$.

Definition $4.10 *$. Let $S$ be a t-conorm and $X \neq \emptyset$. A function $\mathcal{U}: \bigcup_{n \in \mathbb{N}} \square_{n}^{X} \rightarrow[0,1]$ is an $S$-supplementarity multi-measure on $[0,1]^{X}$ if it satisfies, for each $n \in \mathbb{N}$ :

u.1 $\left.\mathcal{U}\left(\mu_{\emptyset}, \cdots\right), \mu_{\emptyset}\right)=1$.

u.2 $\mathcal{U}\left(\mu_{1}, \ldots, \mu_{n}\right)=0$ for all $\left\{\mu_{1}, \ldots, \mu_{n}\right\} \subset[0,1]^{X}$ such that $S\left(\mu_{1}, \ldots, \mu_{n}\right)=\mu_{X}$.

u.3 $\mathcal{U}\left(\mu_{1}, \ldots, \mu_{n}\right)=\mathcal{U}\left(\mu_{\pi(1)}, \ldots, \mu_{\pi(n)}\right)$ holds for all $\pi \in S_{n}$ and $\mu_{1}, \ldots, \mu_{n} \in[0,1]^{X}$.

u.4 Given $\mu_{1}, \ldots, \mu_{n}, \sigma_{1}, \ldots, \sigma_{n} \in[0,1]^{X}$, if $\mu_{i} \leqslant \sigma_{i}$ holds for all $i \in\{1, \ldots, n\}$, then $\mathcal{U}\left(\sigma_{1}, \ldots, \sigma_{n}\right) \leqslant \mathcal{U}\left(\mu_{1}, \ldots, \mu_{n}\right)$.

u.5 $\mathcal{U}\left(\mu_{1}, \ldots, \mu_{n+1}\right) \leqslant \mathcal{U}\left(\mu_{1}, \ldots, \mu_{n}\right)$ holds for all $n \in \mathbb{N}$ and $\mu_{1}, \ldots, \mu_{n+1} \in[0,1]^{X}$.

If $\mathcal{U}$ satisfies axioms $\mathrm{u} .1-\mathrm{u} .4$ for some fixed $n \in \mathbb{N}$, we say that $\mathcal{U}$ is a $U$-unsupplementarity $n$-measure on $[0,1]^{X}$. If $n=2$ we simply say that $\mathcal{S}$ is a $U$-unsupplementarity measure.

Following from Proposition 2.10, we can prove the next result which is a certain extend dually analogous to Proposition 4.2.

Proposition 4.11. Let $X \neq \emptyset$. If $T$ is a t-norm, $S$ and $S_{0}$ are $t$-conorms and $m^{\prime}:[0,1]^{X} \rightarrow[0,1]$ is a fuzzy measure on $\left([0,1]^{X}, \geqslant\right)$, then the functions $\mathcal{U}_{T * m^{\prime}}, \mathcal{U}_{m^{\prime} * S}: \bigcup_{n \in \mathbb{N}} \|_{n}^{X} \rightarrow[0,1]$ defined, for each $\left(\mu_{1}, \ldots, \mu_{n}\right) \in \mathbb{a}_{n}^{X}$ and $n \in \mathbb{N}$, by

$$
\begin{aligned}
& \mathcal{U}_{T+m^{t}}\left(\mu_{1}, \ldots, \mu_{n}\right)= \begin{cases}0 & \text { if } S_{0}\left(\mu_{1}, \ldots, \mu_{n}\right)=\mu_{X} \\
T\left(m^{\prime}\left(\mu_{1}\right), \ldots, m^{\prime}\left(\mu_{n}\right)\right) & \text { otherwise }\end{cases} \\
& \mathcal{U}_{m^{\prime} * S}\left(\mu_{1}, \ldots, \mu_{n}\right)= \begin{cases}0 & \text { if } S_{0}\left(\mu_{1}, \ldots, \mu_{n}\right)=\mu_{X} \\
m^{\prime}\left(S\left(\mu_{1}, \ldots, \mu_{n}\right)\right) & \text { otherwise }\end{cases}
\end{aligned}
$$

are $S_{0}$-unsupplementarity multi-measures on $[0,1]^{X}$.

\section{Remark 4.12. Observe that:}

1. It does not make sense to replace the t-norm $T$ with a t-conorm $S$ in the definition of $\mathcal{U}_{T * m^{\prime}}$, because $S\left(m^{\prime}\left(\mu_{1}\right), \ldots, m^{\prime}\left(\mu_{n}\right)\right)$ $\leqslant S\left(m^{\prime}\left(\mu_{1}\right), \ldots, m^{\prime}\left(\mu_{n}\right), m^{\prime}\left(\mu_{n+1}\right)\right)$, and so axiom $u .5$ is not satisfied.

2. As $T$ is $n$-decreasing and $m^{\prime}$ reverses the order, then $m^{\prime} \circ T$ is $n$ increasing. Thus, it is meaningless to replace the t-conorm $S$ with a t-norm $T$ in the definition of $\mathcal{U}_{m^{\prime}+s}$.

As before, we can replace $T$ and $S$ by the $\mathbf{t}$-norms and $\mathrm{t}$-conorms, respectively, conjugated with the basic triangular norms and conorms in Proposition 4.11.

As in the case of supplementarity, if $S_{L}^{\varphi}$ is the t-conorm $\varphi$-conjugated with the Lukasiewicz $\mathrm{t}$-conorm, taking into account that $S_{L}^{\varphi}\left(\mu_{1}, \ldots, \mu_{n}\right)=\mu_{X}$ if and only if $\sum_{i=1}^{n} \varphi\left(\mu_{i}(x)\right) \geqslant 1$ for all $x \in X$, we can use the difference between $\sum_{i=1}^{n} \varphi\left(\mu_{i}(x)\right)$ and 1 to assign degrees of $S_{L}^{\varphi}$-unsupplementarity. So, we can prove the following result.

Proposition 4.13. Let $X \neq \emptyset$ and $\varphi \in \mathcal{A}([0,1])$. The function $\mathcal{U}_{L}^{\varphi}: \bigcup_{n \in \mathbb{N}} \mathbb{N}_{n}^{X} \rightarrow[0,1]$ defined for each $\left(\mu_{1}, \ldots, \mu_{n}\right) \in \square_{n}^{X}$ by

$\mathcal{U}_{L}^{\varphi}\left(\mu_{1}, \ldots, \mu_{n}\right)=\max \left\{0,1-\inf _{x \in X} \sum_{i=1}^{n} \varphi\left(\mu_{i}(x)\right)\right\}$

is an $S_{L}^{\varphi}$-unsupplementarity multi-measure on $[0,1]^{X}$. 


\section{Some relations among the four types of multi-measures}

Now we show some basic relations among the multi-measures introduced in Sections 3 and 4. To do this, we consider a t-norm $T$ and a t-conorm $S$ that are dual operators with respect to a strong negation $N$, that is, $S(a, b)=N(T(N(a), \quad N(b)))$ or $T(a, b)=$ $N(S(N(a), N(b)))$ for all $a, b \in[0,1]$.

Proposition 5.1. Given a strong negation $N$, let $T$ be a t-norm and $S$ be its $N$-dual $t$-conorm. If $X \neq \emptyset$, then:

1. If $\mathcal{C}$ is a T-compatibility multi-measure on $[0,1]^{X}$, then the function $\mathcal{U}: \cup_{n \in \mathbb{N}} \mathbb{Q}_{n}^{X} \rightarrow[0,1]$, defined for each $\left(\mu_{1}, \ldots, \mu_{n}\right) \in \square_{n}^{X}$ by

$$
\mathcal{U}\left(\mu_{1}, \ldots, \mu_{n}\right)=\mathcal{C}\left(N \circ \mu_{1}, \ldots, N \circ \mu_{n}\right),
$$

is an $S$-unsupplementarity multi-measure on $[0,1]^{X}$.

2. If $\mathcal{U}$ is an S-supplementarity multi-measure on $[0,1]^{X}$, then the function $\mathcal{C}: \cup_{n \in \mathbb{N}} \square_{n}^{X} \rightarrow[0,1]$, defined for each $\left(\mu_{1}, \ldots, \mu_{n}\right) \in \mathbb{1}_{n}^{X}$ by

$$
\mathcal{C}\left(\mu_{1}, \ldots, \mu_{n}\right)=\mathcal{U}\left(N \circ \mu_{1}, \ldots, N \circ \mu_{n}\right),
$$

is a T-compatibility multi-measure on $[0,1]^{X}$.

Proof. We give the proof only for the first case because the second is similar. Let us check the axioms.

(u.1) $\left.\left.\mathcal{U}\left(\mu_{\mathfrak{\emptyset}},,^{n)}, \mu_{\mathfrak{\emptyset}}\right)=\mathcal{C}\left(N \circ \mu_{\mathfrak{\emptyset}},,{ }^{n}\right), N \circ \mu_{\mathfrak{\emptyset}}\right)=\mathcal{C}\left(\mu_{X},{ }^{n}\right), \mu_{X}\right)=1 \quad$ for each $n \in \mathbb{N}$.

(u.2) Let $\left\{\mu_{1}, \ldots, \mu_{n}\right\} \subset[0,1]^{X}$ such that $S\left(\mu_{1}, \ldots, \mu_{n}\right)=\mu_{X}$. This equality is equivalent to

$$
T\left(N \circ \mu_{1}, \ldots, N \circ \mu_{n}\right)=N \circ \mu_{X}=\mu_{\emptyset},
$$

since $S$ is the t-conorm $N$-dual of $T$. Then, from axiom c.2 of compatibility multi-measures, we obtain

$$
\mathcal{U}\left(\mu_{1}, \ldots, \mu_{n}\right)=\mathcal{C}\left(N \circ \mu_{1}, \ldots, N \circ \mu_{n}\right)=0 .
$$

(u.3) As $\mathcal{C}$ is symmetric, it follows that $\mathcal{U}$ is symmetric.

(u.4) If $\mu_{1}, \ldots, \mu_{n}, \sigma_{1}, \ldots, \sigma_{n} \in[0,1]^{X}$ satisfy $\mu_{i} \leqslant \sigma_{i}$, for all $i \in$ $\{1, \ldots, n\}$, then $N \circ \sigma_{i} \leqslant N \circ \mu_{i}$. Thus, from axiom c. 4 we have

$$
\begin{aligned}
\mathcal{U}\left(\sigma_{1}, \ldots, \sigma_{n}\right) & =\mathcal{C}\left(N \circ \sigma_{1}, \ldots, N \circ \sigma_{n}\right) \leqslant \mathcal{C}\left(N \circ \mu_{1}, \ldots, N \circ \mu_{n}\right) \\
& =\mathcal{U}\left(\mu_{1}, \ldots, \mu_{n}\right) .
\end{aligned}
$$

u.5) Taking into account axiom c.5, we have

$$
\begin{aligned}
\mathcal{U}\left(\mu_{1}, \ldots, \mu_{n+1}\right) & =\mathcal{C}\left(N \circ \mu_{1}, \ldots, N \circ \mu_{n}, N \circ \mu_{n+1}\right) \\
& \leqslant \mathcal{C}\left(N \circ \mu_{1}, \ldots, N \circ \mu_{n}\right)=\mathcal{U}\left(\mu_{1}, \ldots, \mu_{n}\right)
\end{aligned}
$$

for all $n \in \mathbb{N}$ and $\mu_{1}, \ldots, \mu_{n+1} \in[0,1]^{X}$.

In the same way as in Proposition 5.1, we can prove the following result.

Proposition 5.2. Given a strong negation $N$, let $T$ be a t-norm and $S$ be its $N$-dual t-conorm. Then,

1. If $\mathcal{I}$ is a T-compatibility multi-measure on $[0,1]^{x}$, then the function $\mathcal{S}: \bigcup_{n \in \mathbb{N}} \square_{n}^{X} \rightarrow[0,1]$, defined for each $\left(\mu_{1}, \ldots, \mu_{n}\right) \in \square_{n}^{X}$ by

$$
\mathcal{S}\left(\mu_{1}, \ldots, \mu_{n}\right)=\mathcal{I}\left(N \circ \mu_{1}, \ldots, N \circ \mu_{n}\right),
$$

is an S-supplementarity multi-measure on $[0,1]^{X}$

2. If $\mathcal{S}$ is an $S$-supplementarity multi-measure on $[0,1]^{X}$, then the function $\mathcal{I}: \bigcup_{n \in \mathbb{N}} \square_{n}^{X} \rightarrow[0,1]$, defined for each $\left(\mu_{1}, \ldots, \mu_{n}\right) \in \mathbb{1}_{n}^{X}$ by

$$
\mathcal{I}\left(\mu_{1}, \ldots, \mu_{n}\right)=\mathcal{S}\left(N \circ \mu_{1}, \ldots, N \circ \mu_{n}\right),
$$

is a T-incompatibility multi-measure on $[0,1]^{X}$.
Table 1

Candidate skill level in Example 1.

\begin{tabular}{lllllll}
\hline & $x_{1}$ & $x_{2}$ & $x_{3}$ & $x_{4}$ & $x_{5}$ & $x_{6}$ \\
\hline $\operatorname{John}\left(\mu_{1}\right)$ & 0.2 & 0.6 & 1.0 & 0.5 & 0.2 & 0.8 \\
Mary $\left(\mu_{2}\right)$ & 0.7 & 0.3 & 0.1 & 0.4 & 0.6 & 0.3 \\
Peter $\left(\mu_{3}\right)$ & 0.2 & 0.9 & 0.9 & 0.8 & 0.4 & 0.5 \\
Alex $\left(\mu_{4}\right)$ & 0.9 & 0.3 & 0.4 & 0.3 & 0.5 & 0.5 \\
\hline
\end{tabular}

\section{An example}

This section shows a simple example to demonstrate the advantage of using the measures introduced in the paper in different real-life situations, and then in expert systems and other areas of computational intelligence.

A fashion company is being set up and needs to select people who have high-level skills in: fashion design $\left(x_{1}\right)$, clothing $\left(x_{2}\right)$, image $\left(x_{3}\right)$, marketing $\left(x_{4}\right)$, social relations $\left(x_{5}\right)$ and leadership $\left(x_{6}\right)$.

The aim is to form a team of people that accounts for all the skills. Table 1 shows four candidates and their respective skill level.

Let us see the extent to which different teams have all skills, that is, let us measure their supplementarity. Consider the supplementarity measures of Corollary 3.13. We will fix $\varphi=i d$ in all cases.

If we regard a team formed by John and Mary, then $\mathcal{S}_{L+\wedge}\left(\mu_{1}, \mu_{2}\right)=0$ as $S_{L}\left(\mu_{1}, \mu_{2}\right) \neq \mu_{X}$ (for example, $\mathcal{S}_{L}\left(\mu_{1}, \mu_{2}\right)\left(x_{1}\right)=$ $\min \{1,0.1+0.7\}=0.8 \neq 1)$. Similarly, we obtain $\mathcal{S}_{L \neq \wedge}\left(\mu_{i}, \mu_{j}\right)=0$ for $i, j \in\{1, \ldots, 4\}$, with $i \neq j$, as $S_{L}\left(\mu_{i}, \mu_{j}\right) \neq \mu_{X}$. The same occurs when considering $\mathcal{S}_{P * \Lambda}$ and $\mathcal{S}_{\mathrm{Max} * \lambda}$ since $S_{\mathrm{Max}} \leqslant S_{P} \leqslant S_{L}$. Thus, the two-member teams are insufficient to meet company expectations.

If we analyze the three-member teams, then we obtain:

- For John, Mary and Peter,

$$
\begin{aligned}
& \mathcal{S}_{L \star \wedge}\left(\mu_{1}, \mu_{2}, \mu_{3}\right)=\min \left\{1, \sum_{i=1}^{3} \inf _{j} \mu_{i}\left(x_{j}\right)\right\}=\min \{1,0.2+0.1+0.2\}=0.5, \\
& \mathcal{S}_{P_{* \wedge}}\left(\mu_{1}, \mu_{2}, \mu_{3}\right)=1-\prod_{i=1}^{3}\left(1-\inf _{j} \mu_{i}\left(x_{j}\right)\right)=1-0.8 \cdot 0.9 \cdot 0.8=0.424, \\
& \mathcal{S}_{\operatorname{Max} \star \wedge}\left(\mu_{1}, \mu_{2}, \mu_{3}\right)=\operatorname{maxinf}_{1 \leqslant i \leqslant 3} \mu_{i}\left(x_{j}\right)=0.2
\end{aligned}
$$

- For John, Mary and Alex,

$$
\begin{aligned}
& \mathcal{S}_{L * \wedge}\left(\mu_{1}, \mu_{2}, \mu_{4}\right)=\min \{1,0.2+0.1+0.3\}=0.6, \\
& \mathcal{S}_{P_{* \wedge}}\left(\mu_{1}, \mu_{2}, \mu_{4}\right)=1-0.8 \cdot 0.9 \cdot 0.7=0.496 \\
& \mathcal{S}_{\mathrm{MaX} * \wedge}\left(\mu_{1}, \mu_{2}, \mu_{4}\right)=0.3 .
\end{aligned}
$$

- For John, Peter and Alex,

$$
\begin{aligned}
& \mathcal{S}_{L * \wedge}\left(\mu_{1}, \mu_{3}, \mu_{4}\right)=\min \{1,0.2+0.2+0.3\}=0.7 \\
& \mathcal{S}_{P_{* \wedge}}\left(\mu_{1}, \mu_{3}, \mu_{4}\right)=1-0.8 \cdot 0.8 \cdot 0.7=0.552 \\
& \mathcal{S}_{\text {Max } \star}\left(\mu_{1}, \mu_{3}, \mu_{4}\right)=0.3 .
\end{aligned}
$$

- For Mary, Peter and Alex,

$$
\begin{aligned}
& \mathcal{S}_{L+\wedge}\left(\mu_{2}, \mu_{3}, \mu_{4}\right)=\min \{1,0.1+0.2+0.3\}=0.6, \\
& \mathcal{S}_{P_{* \wedge}}\left(\mu_{2}, \mu_{3}, \mu_{4}\right)=1-0.9 \cdot 0.8 \cdot 0.7=0.496 \\
& \mathcal{S}_{\operatorname{Max} \star \wedge}\left(\mu_{2}, \mu_{3}, \mu_{4}\right)=0.3
\end{aligned}
$$

So, the team formed by John, Peter and Alex appears to be the most competent of the three-member teams.

Finally, if we need more supplementarity, that is, higher levels of all the skills, we can consider all four candidates at once:

$\mathcal{S}_{L+\wedge}\left(\mu_{1}, \mu_{2}, \mu_{3}, \mu_{4}\right)=\min \{1,0.2+0.1+0.2+0.3\}=0.8$

$\mathcal{S}_{P_{* \wedge}}\left(\mu_{1}, \mu_{2}, \mu_{3}, \mu_{4}\right)=1-0.8 \cdot 0.9 \cdot 0.8 \cdot 0.7=0.5968$,

$\mathcal{S}_{\mathrm{MlaX} * \wedge}\left(\mu_{1}, \mu_{2}, \mu_{3}, \mu_{4}\right)=0.3$ 
In this way, if we need a fixed degree of supplementarity, new candidates can be added so that this is achieved. Note that the first measure is more sensitive to the changes, and hence could be more significant.

If the company can employ no more than two of the candidates, even if not all expectations are met, measures of unsupplementarity can help to discern which is the best team. So, considering the measure $\mathcal{U}_{L}$, we have:

$\mathcal{U}_{L}\left(\mu_{1}, \mu_{2}\right)=\max \{0,1-\min \{0.9,0.9,1.1,0.9,0.8,1.1\}\}=\max \{0,0.2\}=0.2$,
$\mathcal{U}_{L}\left(\mu_{1}, \mu_{3}\right)=\max \{0,1-\min \{0.4,1.5,1.9,1.3,0.6,1.3\}\}=\max \{0,0.6\}=0.6$,
$\mathcal{U}_{L}\left(\mu_{1}, \mu_{4}\right)=\max \{0,1-\min \{1.1,0.9,1.4,1.8,0.7,1.3\}\}=\max \{0,0.7\}=0.3$,
$\mathcal{U}_{L}\left(\mu_{2}, \mu_{3}\right)=\max \{0,1-\min \{0.9,1.2,1.0,1.2,1.0,0.8\}\}=\max \{0,0.2\}=0.2$,
$\mathcal{U}_{L}\left(\mu_{2}, \mu_{4}\right)=\max \{0,1-\min \{1.6,0.6,0.5,0.7,1.1,0.8\}\}=\max \{0,0.5\}=0.5$,
$\mathcal{U}_{L}\left(\mu_{3}, \mu_{4}\right)=\max \{0,1-\min \{1.1,1.2,1.3,1.1,0.9,1.0\}\}=\max \{0,0.1\}=0.1$.

So, the best two-member team should be the one with the least unsupplementarity, and is hence the team formed by Peter and Alex.

\section{Conclusions}

In this paper, first, we introduce an axiomatic model of multimeasures on a bounded lattice, illustrating some examples for lattices of classical sets. We also set out two methods for building multi-measures. Next we study two types of multi-measures in the particular case of a lattice of fuzzy sets $\left([0,1]^{X}, \leqslant\right)$, namely, $\mathrm{T}$-compability and S-supplementarity measures. Afterwards we address two kinds of multi-measures on $\left([0,1]^{X}, \geqslant\right)$, T-incompatibility and S-unsupplementarity, which are to a certain extent opposite to the former. Both assume the classical case. Moreover we establish some relationships between given pairs of multi-measures.

The main novelty of this axiomatic model is that it provides functions that measure the degree to which a property is verified by a subset of elements of any bounded lattice, regardless of the cardinality of that subset. This contrasts with most of the measures proposed in the literature that can only be used to measure one element or to compare two elements. In particular, our proposal extends the model of aggregation functions which refer to the lattice $([0,1]$, $\leqslant)$ only.

The example given in Section 6 applies the presented functions to a particular decision-making problem. It clearly illustrates the advantage of using these functions, which are able to discern the extend to which a property is satisfied when new elements are added for comparison.

We intend to pursue two main lines of research in the future. On the theoretical side, we will further study the continuity of the introduced measures and other structural properties, and develop analogous measures in the area of Atanassov's fuzzy sets. On the practical side, we will use these measures in approximate reasoning, clustering, expert systems, patter recognition, etc.

\section{Acknowledgement}

This work has been partially supported by DGI (Spain) Projects TIN2011-29827-C02-01, TIN2009-07901, MTM 2009-10962, TIN2012-32482 and by UPM-CAM.

\section{References}

[1] C. Alsina, M. Frank, B. Schweizer, Associative Functions: Triangular Norms and Copulas, World Scientific, Singapore, 2006.

[2] R.C. Bassanezi, G.H. Greco, Functionals representable by fuzzy measures, Journal of Mathematical Analysis and Applications 133 (1988) 44-56.

[3] G. Beliakov, A. Pradera, T. Calvo, Aggregation Functions: A Guide for Practitioners, Springer-Verlag, Berlin, 2007.

[4] D. Ben-Arieh, Z. Chen, Linguistic-lables aggregation and consensus measure for autocratic decision making, using group recommendations, IEEE Transactions on Systems, Man and Cybernetics-Part A: Systems and Humans 36 (3) (2006) 558-568.

[5] G. Birkhoff, Lattice Theory, American Mathematical Society, Providence, 1940

[6] T.S. Blyth, Lattices and Ordered Algebraic Structures, Springer-Verlag, London, 2005.

[7] É. Borel Leçons sur la thêorie des fonctions (in French), Gauthier-Víllars, Paris, 1898.

[8] H. Bustince, J. Fernăndez, R. Mesiar, J. Montero, R. Orduna, Overlap functions, Nonlinear Analysis 72 (2010) 1488-1499.

[9] T. Calvo, A. Kolesarová, M. Komorníková, R. Mesiar, Aggregation operators: properties, in: T. Calvo, R. Mesiar, G. Mayor (Eds.), Classes and Construction Methods in Aggregation Operators: New Trends and Applications, PhysicaVerlag, Heilderberg, 2002, pp. 1-104.

[10] E.E. Castiñeira, T. Calvo, S. Cubillo, Multi-arguments fuzzy measures on some special lattices, in: Proc. of 11th International Conference on Computational and Mathematical Methods in Science and Engineering, (CMMS-2011), Benidorm, Spain, 2011, pp. 319-330.

[11] E. Castiñeira, S. Cubillo, W. Montilla, Measuring incompatibility between Atanassov's intuitionistic fuzzy sets, Information Sciences 180 (2011) 820833.

[12] E.E. Castiñeira, C. Torres-Blanc, S. Cubillo, Measuring contradiction on A-IFS defined in Finite Universes, Knowledge-Based Systems 24 (2011) 1297-1309.

[13] G. Choquet, Theory of capacities, Annales de l'Institut Fourier 5 (1954) 131 295.

[14] S. Cubillo, E. Castiñeira, Measuring contradiction in Fuzzy Logic, International Journal of General Systems 34 (1) (2005) 39-59.

[15] S. Cubillo, E.E. Castiñeira, W. Montilla, Supplementarity measures on fuzzy sets, in: Proc. of 7 th Conference of the European Society for Fuzzy Logic and Technology (EUSFLAT-LFA 2011), Aix-Les-Bains, France, pp. 897-903.

[16] A.P. Dempster, Upper and lower probabilities induced by multi-valued mapping, Annals of Mathematical Statistics 38 (1967) 325-339.

[17] D. Dennenberg, Non-Additive Measure and Integral, Kluwer Academic Publisher, Dordrecht, 1994.

[18] D. Dubois, H. Prade, Evidence measures based on fuzzy information, Automatica 21 (1985) 547-562.

[19] D. Dubois, H. Prade, Possibility Theory, Plenum Press, New York, 1988 (translated from the French original published in 1985).

[20] M. Grabisch, J.L. Marichal, R. Mesiar, E. Pap, Aggregation Functions, Cambridge University Press, Cambridge, 2009.

[21] M. Grabish, T. Murofushi, M. Sugeno (Eds.), Fuzzy Measures and Integrals. Theory and Applications, Physica-Verlag, Heidelberg, 2000.

[22] G.H. Greco, Fuzzy integrals and fuzzy measures with their values in complete lattices, Journal of Mathematical Analysis and Applications 126 (1987) 594603.

[23] F. Herrera, E. Herrera-Viedma, Linguistic decision analysis: steps for solving decision problems under linguistic information, Fuzzy Sets and Systems 115 (1) (2000) 67-82.

[24] J. Jacas, J. Recasens, Fuzzy T-transitive relations: eigenvectors and generators, Fuzzy Sets \& Systems 72 (1995) 147-154.

[25] V. G Kaburlasos, V. Petridis, Fuzzy Lattice Neurocomputing (FLN): a novel conectionist scheme for versatile learning and decision making by clustering, International Journal of Computers and Their Applications 4 (3) (1997) 31-43.

[26] V. G Kaburlasos, Towards a unified modelling and knowledge representationbased on lattice theory-computational intelligence and soft computing applications, Studies on Computational Intelligence, vol. 27, Springer, HeidelbergGermany, 2006

[27] V. G Kaburlasos, A. Kehagias, Novel fuzzy inference system (FIS) analysis and design based on lattice theory, Part I: working principles, International Journal of General Systems 35 (1) (2006) 45-67.

[28] Vassilis G. Kaburlasos, Joannis N. Athanasiadis, Pericles A. Mitkas, FLR classifier and its application for ambient ozone estimation, Int. Journal on Approximate Reasoning 45 (2007) 152-188.

[29] E.P. Klement, Fuzzy $\sigma$-algebras and fuzzy measurrable functions, Fuzzy Sets and Systems 4 (1980) 83-93.

[30] E.P. Klement, R. Mesiat, E. Pap, Triangulat Norms, Kluwer Academic Publisher, Dordrecht, 2000.

[31] E.P. Klement, S. Weber, Generalized measures, Fuzzy Sets and Systems 40 (1991) 375-394.

[32] A.N. Kolmogorov, Foundations of the Theory of Probability, Chelsea, New York, 1950.

[33] R. Kruse, On the construction of fuzzy measures, Fuzzy Sets and Systems 8 (1982) 67-74.

[34] H. Lebesgue, Measure and the Integral, Holden-Day, San Francisco, 1966.

[35] H. Liu, S. Xiong, Z. Fang, Fl-GrCCA: a granular computing classification algorithm based on fuzzy lattices, Computers and Mathematics with Applications 61 (1) (2011) 138-147.

[36] X. Liu, Futher discussion on convergence theorems for seminormed fuzzy integral and semiconormed fuzzy integrals, Fuzzy Sets and Systems 55 (1993) 219-226.

[37] X. Liu, G. Zhang, Lattice-valued fuzzy measure and lattice-valued fuzzy integral, theorems for seminormed fuzzy integral and semiconormed fuzzy integrals, Fuzzy Sets and Systems 62 (1994) 319-332.

[38] R. Mesiar, A. Mesiarová, Fuzzy integrals, in: V. Torra, Y. Narukawa, A. Valls (Eds.), Modelling Decisions For Artificial Intelligence, LNAI 3131, Springer, Berlin, 2004, pp. 7-14. 
[39] R. Mesiar, A. Mesiarová, L. Valásáková, Generated universal fuzzy measures, in V. Torra, Y. Narukawa, A. Valls, J. Domingo-Ferre (Eds.), Modelling Decisions For Artificial Intelligence, LNAI 3885, Springer, Berlin, 2006, pp. 191-202.

[40] R. Mesiar, A. Mesiarová-Zemánková, L. Valásková, Basic generated universal fuzzy measures, International Journal of Approximate Reasonning 46 (2007) 447-457.

[41] R. Mesiar, M. Komorniková, Aggregation functions on bounded poset, in: C. Cornelis et al. (Eds.), 35 Years of Fuzzy set Theory, STUDFUZZ 261, SpringerVerlag, 2010, pp. 3-17.

[42] E. Pap (Ed.), Handbook of Measure Theory, North Holland/Elsevier, Amsterdam, Boston, 2002

[43] Z. Qiao, On fuzzy measure and fuzzy integrals on fuzzy sets, Fuzzy Sets and Systems 37 (1990) 77-92.

[44] Z. Qiao, Fuzzy integrals on L-fuzzy sets, Fuzzy Sets and Systems 38 (1990) 6179.

[45] D. Ralescu, G. Adams, The fuzzy integral, Journal of Mathematical Analysis and Applications 75 (1980) 562-570.

[46] G.X. Ritter, G. Urcid, Lattice algebra approach to single-neuron computation, IEEE Transactions on Neural Networks 14 (2) (2003) 282-295.

[47] B. Schweizer, A. Sklar, Associative functions and statistical triangle inequalities, Publicationes Mathematicae-Debrecen 8 (1961) 169-186.
[48] G. Shafer, A Mathematical Theory of Evidence, Princeton University Press, Princeton, 1976.

[49] M. Sugeno, Theory of Fuzzy Integrals and its Applications, Ph.D. Dissertation, Tokyo Institute of Technology, 1974.

[50] V. Torra, Y. Narukawa, Modelling Decisions: Information Fusion and Aggregation Operators, Springer-Verlag, Berlin, 2007.

[51] E. Trillas, Sobre funciones de negación en los conjuntos difusos (in Spanish), Stochastica 3 (1) (1979) 47-60 (Reprinted (English version) in Advances of Fuzzy Logic. Eds. S. Barro et alri (Universidad de Santiago de Compostela), (1998) 31-43)

[52] Z. Wang, Asymptotic structural characteristics of fuzzy measure and their applications, Fuzzy Sets and Systems 16 (1985) 277-290.

[53] Y. Jamshidi Khezeli, H. Nezamabadi, Fuzzy lattice reasoning for pattern classification using a new positive valuation functions, Advances in Fuzzy Systems 2012 (2012), http://dx.doi.org/10.1155/2012/206121.

[54] L.A. Zadeh, Fuzzy sets, Information and Control 8 (1965) 338-353.

[55] L.A. Zadeh, Similarity relations and fuzzy orderings, Information Sciences 3 (1971) 177-200.

[56] L.A. Zadeh, Fuzzy sets as a basis for a theory of possibility, Fuzzy Sets and Systems 1 (1978) 3-28. 